\title{
Kaon $B$ parameter with the Wilson quark action using chiral Ward identities
}

\author{
S. Aoki,${ }^{1}$ M. Fukugita, ${ }^{2}$ S. Hashimoto,${ }^{3}$ N. Ishizuka, ${ }^{1,4}$ Y. Iwasaki, ${ }^{1,4}$ K. Kanaya,,${ }^{1,4}$ Y. Kuramashi, ${ }^{5, *}$ M. Okawa, ${ }^{6}$ \\ A. Ukawa, ${ }^{1,4}$ and T. Yoshié ${ }^{1,4}$ \\ (JLQCD Collaboration) \\ ${ }^{1}$ Institute of Physics, University of Tsukuba, Tsukuba, Ibaraki 305-8571, Japan \\ ${ }^{2}$ Institute for Cosmic Ray Research, University of Tokyo, Tanashi, Tokyo 188-8502, Japan \\ ${ }^{3}$ Computing Research Center, High Energy Accelerator Research Organization (KEK), Tsukuba, Ibaraki 305-0801, Japan \\ ${ }^{4}$ Center for Computational Physics, University of Tsukuba, Tsukuba, Ibaraki 305-8577, Japan \\ ${ }^{5}$ Department of Physics, Washington University, St. Louis, Missouri 63130 \\ ${ }^{6}$ Institute of Particle and Nuclear Studies, High Energy Accelerator Research Organization (KEK), Tsukuba, Ibaraki 305-0801, Japan
}

(Received 22 January 1999; published 8 July 1999)

\begin{abstract}
A lattice QCD calculation of the kaon $B$ parameter $B_{K}$ is carried out with the Wilson quark action in the quenched approximation at $\beta=6 / g^{2}=5.9-6.5$. The mixing problem of the $\Delta s=2$ four-quark operators is solved nonperturbatively with full use of chiral Ward identities employing four external quarks with an equal off-shell momentum in the Landau gauge. This method, without invoking any effective theory, enables us to construct the weak four-quark operators exhibiting good chiral behavior. Our results for $B_{K}$ with the nonperturbative mixing coefficients show small scaling violation beyond the lattice cutoff $a^{-1} \sim 2.5 \mathrm{GeV}$. Our estimate concludes $B_{K}(\mathrm{NDR}, 2 \mathrm{GeV})=0.69(7)$ at $a^{-1}=2.7-4.3 \mathrm{GeV}$, which agrees with the value obtained with the Kogut-Susskind quark action. For comparison we also calculate $B_{K}$ with one-loop perturbative mixing coefficients. While this yields incorrect values at finite lattice spacing, a linear extrapolation to the continuum limit as a function of $a$ leads to a result consistent with those obtained with the Ward identity method. [S0556-2821(99)05913-5]
\end{abstract}

PACS number(s): 12.38.Gc, 11.15.Ha, 13.75.Jz

\section{INTRODUCTION}

The kaon $B$ parameter defined as a ratio

$$
B_{K}=\frac{\left\langle\bar{K}^{0}\left|\bar{s} \gamma_{\mu}\left(1-\gamma_{5}\right) d \cdot \bar{s} \gamma_{\mu}\left(1-\gamma_{5}\right) d\right| K^{0}\right\rangle}{(8 / 3)\left\langle\bar{K}^{0}\left|\bar{s} \gamma_{\mu} \gamma_{5} d\right| 0\right\rangle\left\langle 0\left|\bar{s} \gamma_{\mu} \gamma_{5} d\right| K^{0}\right\rangle}
$$

is one of the fundamental weak matrix elements which have to be determined theoretically for deducing the $C P$ violation phase of the Cabibbo-Kobayashi-Maskawa matrix from experiments. Lattice QCD calculation is expected to evaluate $B_{K}$ precisely incorporating the long-distance effects of QCD. Much effort has been devoted over the years to this purpose, using both the Wilson and the Kogut-Susskind (KS) quark actions. Successful calculations of $B_{K}$ have been achieved so far with the KS quark action, taking advantage of the correct chiral behavior of the matrix element ensured by U(1) chiral symmetry $[1,2]$, while studies with the Wilson quark action are rather stagnant. There are two purposes for us to try to advance the calculations of $B_{K}$ with the Wilson action. One of them is to verify the consistency between the Wilson and the KS results, which would give full credit to the lattice QCD calculation. The other is an application to the heavylight system for which the interpretation of flavor quantum numbers with the KS action is difficult.

\footnotetext{
* On leave from Institute of Particle and Nuclear Studies, High Energy Accelerator Research Organization (KEK), Tsukuba, Ibaraki 305-0801, Japan.
}

An annoying defect of the Wilson quark action is explicit breaking of chiral symmetry at finite lattice spacing. For the calculation of $B_{K}$ the problem appears as a nontrivial mixing of the weak $\Delta s=2$ four-quark operator of purely left handed chirality with those of mixed left-right chirality. Early studies showed that the mixing problem is not adequately treated by perturbation theory, leading to an "incorrect answer" for the matrix element [3]. Most calculations of $B_{K}$ have then tried to solve the mixing problem nonperturbatively with the aid of chiral perturbation theory [4,5], and have succeeded in giving reasonable estimates for $B_{K}$. This method, however, is not promising from a point of view to control systematic errors, since it contains large uncertainties from higher order effects of chiral perturbation theory which survive even in the continuum limit.

An essential step toward a precise determination of $B_{K}$ is to control the operator mixing nonperturbatively without resort to any effective theories. The failure of the perturbative approach suggests that higher order corrections in terms of the coupling constant might be large in the mixing coefficients. Presence of large corrections in powers of the lattice spacing $a$ in the mixing coefficients is also a possibility. In order to deal with this problem, the Rome group has proposed the method of nonperturbative renormalization (NPR) [6]. Numerical results based on this approach show an improvement of the chiral behavior of the $\Delta s=2$ operator [7].

In this paper we propose an alternative nonperturbative method to solve the operator mixing problem which is based on the use of chiral Ward identities [8]. This method fully incorporates the chiral properties of the Wilson action explicitly. We also reexamine the question if perturbative mix- 
ing coefficients lead to erroneous results for $B_{K}$ in the continuum limit. Our simulations have been made within quenched QCD at $\beta=5.9-6.5$ keeping the physical spatial size approximately constant at $2.4 \mathrm{fm}$. The chief findings of our calculation have already been presented in Ref. [9] and we give in this article a detailed description of the implementation of our method and the results of our analyses.

This paper is organized as follows. In Sec. II we describe the formalism of our nonperturbative method to determine the mixing coefficients for four-quark operators based on the use of chiral Ward identities. The perturbative expressions for the overall renormalization factors are also given. In Sec. III we present our data sets and give a description of the calculational procedure for $B_{K}$. Results for the mixing coefficients are given in Sec. IV, where we compare our results with those for the NPR method. The overall renormalization factors determined by the NPR method are compared with those obtained by the perturbative one in Sec. V. In Sec. VI we examine the chiral properties of the four-quark operators constructed with the Ward identity method. The final results for $B_{K}$ are presented in Sec. VII. Through Secs. IV-VII we also present results with the perturbative method for comparative purposes. Our conclusions are summarized in Sec. VIII.

\section{FORMULATION OF THE METHOD}

\section{A. Determination of the mixing coefficients}

We first derive the generic form of the chiral Ward identities in a standard manner [8]. The Wilson quark action is defined by

$$
\begin{aligned}
S_{W}= & -\frac{1}{2} \sum_{x, \mu}\left[\bar{\psi}(x)\left(1-\gamma_{\mu}\right) U_{\mu}(x) \psi(x+\hat{\mu})\right. \\
& \left.+\bar{\psi}(x+\hat{\mu})\left(1+\gamma_{\mu}\right) U_{\mu}^{\dagger}(x) \psi(x)\right] \\
& +\sum_{x} \bar{\psi}(x)\left(m_{0}+4\right) \psi(x),
\end{aligned}
$$

where $\psi=(u, d, s)$ represents the up, down, and strange quark fields. The conventional hopping parameter is given by

$$
K=\frac{1}{2 m_{0}+8} .
$$

Under the flavor SU(3) chiral variation defined by

$$
\begin{aligned}
& \delta^{a} \psi(x)=i \frac{\lambda^{a}}{2} \gamma_{5} \psi(x), \\
& \delta^{a} \bar{\psi}(x)=\bar{\psi}(x) i \frac{\lambda^{a}}{2} \gamma_{5},
\end{aligned}
$$

with $\lambda^{a}(a=1, \ldots, 8)$ the flavor matrices normalized as $\operatorname{Tr}\left(\lambda^{a} \lambda^{b}\right)=2 \delta^{a b}$, the naive Ward identity that follows from the Noether procedure takes the form

$$
\begin{aligned}
\left\langle\nabla_{\mu} A_{\mu}^{\mathrm{ext}, a}(x) \mathcal{O}\left(x_{1}, \ldots, x_{n}\right)\right\rangle= & 2 m_{0}\left\langle P^{a}(x) \mathcal{O}\left(x_{1}, \ldots, x_{n}\right)\right\rangle \\
& +\left\langle X^{a}(x) \mathcal{O}\left(x_{1}, \ldots, x_{n}\right)\right\rangle \\
& +i\left\langle\delta^{a} \mathcal{O}\left(x_{1}, \ldots, x_{n}\right)\right\rangle,
\end{aligned}
$$

where the pseudoscalar density $P^{a}$, the extended axial vector current $A_{\mu}^{\mathrm{ext}, a}$ and its divergence are defined by

$$
\begin{aligned}
P^{a}(x)= & \bar{\psi}(x) \frac{\lambda^{a}}{2} \gamma_{5} \psi(x) \\
A_{\mu}^{\mathrm{ext}, a}(x)= & \frac{1}{2}\left[\bar{\psi}(x) \frac{\lambda^{a}}{2} \gamma_{\mu} \gamma_{5} U_{\mu}(x) \psi(x+\hat{\mu})+\bar{\psi}(x+\hat{\mu})\right. \\
& \left.\times \frac{\lambda^{a}}{2} \gamma_{\mu} \gamma_{5} U_{\mu}^{\dagger}(x) \psi(x)\right] \\
\nabla_{\mu} A_{\mu}^{\mathrm{ext}, a}(x)= & \sum_{\mu}\left[A_{\mu}^{\mathrm{ext}, a}(x)-A_{\mu}^{\mathrm{ext}, a}(x-\hat{\mu})\right]
\end{aligned}
$$

and the $X^{a}$ term is given by

$$
\begin{aligned}
X^{a}(x)= & -\frac{1}{2} \sum_{\mu}\left[\bar{\psi}(x) \frac{\lambda^{a}}{2} \gamma_{5} U_{\mu}(x) \psi(x+\hat{\mu})+\bar{\psi}(x+\hat{\mu})\right. \\
& \left.\times \frac{\lambda^{a}}{2} \gamma_{5} U_{\mu}^{\dagger}(x) \psi(x)+(x \rightarrow x-\hat{\mu})\right] \\
& +8 \bar{\psi}(x) \frac{\lambda^{a}}{2} \gamma_{5} \psi(x) .
\end{aligned}
$$

The $X^{a}$ term mixes with $P^{a}$ and $\nabla_{\mu} A_{\mu}^{a}$ under renormalization. Thus we write

$$
X^{a}(x)=\bar{X}^{a}(x)-2 \delta m_{0} P^{a}(x)-\left(Z_{A}^{\mathrm{ext}, a}-1\right) \nabla_{\mu} A_{\mu}^{\mathrm{ext}, a}(x),
$$

where $\bar{X}^{a}(x)$ should satisfy the following.

(i) On-shell matrix elements vanish in the continuum limit:

$$
\left\langle\alpha\left|\bar{X}^{a}(x)\right| \beta\right\rangle=O(a) .
$$

(ii) Off-shell Green functions have only contact terms up to terms of $O(a)$ :

$$
\begin{aligned}
\left\langle\bar{X}^{a}(x) \mathcal{O}\left(x_{1}, \ldots, x_{n}\right)\right\rangle= & \sum_{i} \delta\left(x-x_{i}\right)\left\langle\mathcal{O}_{i}^{\prime a}\left(x_{1}, \ldots, x_{n}\right)\right\rangle \\
& +O(a)
\end{aligned}
$$

Defining the renormalized axial vector current by

$$
\hat{A}_{\mu}^{\mathrm{ext}, a}(x)=Z_{A}^{\mathrm{ext}} A_{\mu}^{\mathrm{ext}, a}(x)
$$

and the renormalized quark mass by

$$
m=m_{0}-\delta m_{0}
$$

the Ward identity takes the following form: 


$$
\begin{aligned}
\left\langle\nabla_{\mu} \hat{A}_{\mu}^{\mathrm{ext}, a}(x) \mathcal{O}\left(x_{1}, \ldots, x_{n}\right)\right\rangle= & 2 m\left\langle P^{a}(x) \mathcal{O}\left(x_{1}, \ldots, x_{n}\right)\right\rangle \\
& +\left\langle\bar{X}^{a}(x) \mathcal{O}\left(x_{1}, \ldots, x_{n}\right)\right\rangle \\
& +i\left\langle\delta^{a} \mathcal{O}\left(x_{1}, \ldots, x_{n}\right)\right\rangle .
\end{aligned}
$$

For finite quark masses it is also useful to take a fourdimensional sum over $x$, which gives

$$
\begin{aligned}
2 m \sum_{x} & \left\langle P^{a}(x) \mathcal{O}\left(x_{1}, \ldots, x_{n}\right)\right\rangle+\sum_{x}\left\langle\bar{X}^{a}(x) \mathcal{O}\left(x_{1}, \ldots, x_{n}\right)\right\rangle \\
& +\sum_{x} i\left\langle\delta^{a} \mathcal{O}\left(x_{1}, \ldots, x_{n}\right)\right\rangle=0 .
\end{aligned}
$$

We note that $\bar{X}^{a}(x)$ in Eqs. (16) and (17) generates only contact terms up to terms of $O(a)$.

Let us consider a set of weak operators in the continuum $\left\{\mathcal{O}_{i}\right\}$ which closes under flavor chiral rotations $\delta^{a} \hat{\mathcal{O}}_{i}$ $=i c_{i j}^{a} \hat{\mathcal{O}}_{j}$. These operators are given by linear combinations of a set of lattice local operators $\left\{\mathcal{O}_{\alpha}\right\}$ as $\hat{\mathcal{O}}_{i}=\Sigma_{\alpha} Z_{i \alpha} \mathcal{O}_{\alpha}$. We choose the mixing coefficients $Z_{i \alpha}$ such that the Green functions of $\left\{\hat{\mathcal{O}}_{i}\right\}$ with quarks in the external states satisfy the chiral Ward identity to $O(a)$. This identity is obtained from Eq. (17):

$$
\begin{aligned}
& -2 \rho_{m} Z_{A}^{\mathrm{ext}} \sum_{x}\left\langle P^{a}(x) \hat{\mathcal{O}}_{i}(0) \prod_{k} \widetilde{\psi}\left(p_{k}\right)\right\rangle \\
& +c_{i j}^{a}\left\langle\hat{\mathcal{O}}_{j}(0) \prod_{k} \widetilde{\psi}\left(p_{k}\right)\right\rangle \\
& -i \sum_{l}\left\langle\hat{\mathcal{O}}_{i}(0) \prod_{k \neq l} \widetilde{\psi}\left(p_{k}\right) \delta^{a} \widetilde{\psi}\left(p_{l}\right)\right\rangle+O(a)=0,
\end{aligned}
$$

where $p_{k}$ is the momentum of the external quark. We note that the first term in Eq. (18) comes from the chiral variation of the Wilson quark action and the third represents the chiral rotation of the external fields. Since the identity (18) is linear in the renormalization constants, the overall renormalization factor cannot be fixed. Furthermore, with quarks in the external states, calculations have to be made in some fixed gauge, e.g., the Landau gauge.

The $O(a)$ term is governed by the typical QCD scale $\Lambda_{\mathrm{QCD}}$ at low external quark momenta, while powers of $p_{k} a$ become the dominant source of cutoff effects as momenta increase. To be able to impose the Ward identity to $O(a)$, we need to restrict the external momenta by the condition $p_{k} \ll 1 / a$ for large momenta. On the other hand, no such bounds exist for small momenta for the validity of the identity itself as long as $\Lambda_{\mathrm{QCD}} a \ll 1$.

The parameter $\rho_{m}=\left(m_{0}-\delta m_{0}\right) / Z_{A}^{\text {ext }}$ in Eq. (18) is determined from the PCAC (partial conservation of axial vector current) relation obtained from an application of the Ward identity (16) for $\mathcal{O}=P^{b}(y)[10]$ :

$$
\begin{aligned}
\left\langle\nabla_{\mu} A_{\mu}^{\mathrm{ext}, a}(x) P^{b}(y)\right\rangle & \\
= & 2 \rho_{m}\left\langle P^{a}(x) P^{b}(y)\right\rangle-\delta(x-y) \\
\quad & \times \frac{1}{Z_{A}^{\mathrm{ext}}}\left\langle\bar{\psi}(y)\left[\frac{1}{3} \delta^{a b} \mathbf{1}+d^{a b c} \frac{\lambda^{c}}{2}\right] \psi(y)\right\rangle+O(a) .
\end{aligned}
$$

For the determination of $Z_{A}^{\text {ext }}$ we employ another Ward identity

$$
\begin{aligned}
& -2 \rho_{m} Z_{A}^{\mathrm{ext}} \sum_{x}\left\langle P^{a}(x) A_{\mu}^{b}(y) V_{\nu}^{c}(z)\right\rangle \\
& =+i f^{a b d} \frac{Z_{V}}{Z_{A}}\left\langle V_{\mu}^{d}(y) V_{\nu}^{c}(z)\right\rangle+i f^{a c d} \frac{Z_{A}}{Z_{V}}\left\langle A_{\mu}^{b}(y) A_{\nu}^{d}(z)\right\rangle \\
& \quad+O(a)
\end{aligned}
$$

where we take $\mathcal{O}=A_{\mu}^{b}(y) V_{\nu}^{c}(z)$ in Eq. (17) [10]. The local axial vector current and the local vector current are defined by

$$
\begin{aligned}
A_{\mu}^{b}(y) & =\bar{\psi}(y) \frac{\lambda^{b}}{2} \gamma_{\mu} \gamma_{5} \psi(y), \\
V_{\nu}^{c}(z) & =\bar{\psi}(z) \frac{\lambda^{c}}{2} \gamma_{\nu} \psi(z),
\end{aligned}
$$

and $Z_{A}$ and $Z_{V}$ are the renormalization factors for $A_{\nu}^{b}(y)$ and $V_{\rho}^{c}(z)$, respectively. The identity (20) can be regarded as a set of equations for $Z_{A}^{\text {ext }}$ and $Z_{A} / Z_{V}$. Two independent equations are obtained from $\mu=\nu=4$ and $\mu=\nu=i(i=1,2,3)$.

The continuum four-quark operator relevant for $B_{K}$ is given by

$$
\hat{\mathcal{O}}_{V V+A A}=\left(\bar{s} \gamma_{\mu} d\right)\left(\bar{s} \gamma_{\mu} d\right)+\left(\bar{s} \gamma_{\mu} \gamma_{5} d\right)\left(\bar{s} \gamma_{\mu} \gamma_{5} d\right)
$$

where the parentheses mean color trace, and the parity violating part of the operator which does not contribute to $B_{K}$ is dropped. To fix the mixing coefficients for the lattice fourquark operators, we may choose a particular SU(3) flavor chiral rotation to be applied for $\hat{\mathcal{O}}_{V V+A A}$. In order to avoid complexities in numerical simulations it is essential to avoid flavor rotations that yield operators which have penguin contractions and hence mix with lower dimension operators. Assuming $\mathrm{SU}(2)$ symmetry $m_{u}=m_{d}$ we employ the $\lambda^{3}$ $=\operatorname{diag}(1,-1,0)$ chiral rotation, under which $\hat{\mathcal{O}}_{V V+A A}$ and $\hat{\mathcal{O}}_{V A}=\left(\bar{s} \gamma_{\mu} d\right)\left(\bar{s} \gamma_{\mu} \gamma_{5} d\right)$ form a minimal closed set of the operators

$$
\begin{gathered}
\delta^{3} \frac{1}{2} \hat{\mathcal{O}}_{V V+A A}=-i \hat{\mathcal{O}}_{V A}, \\
\delta^{3} \hat{\mathcal{O}}_{V A}=-i \frac{1}{2} \hat{\mathcal{O}}_{V V+A A} .
\end{gathered}
$$


Since $\hat{\mathcal{O}}_{V V+A A}$ and $\hat{\mathcal{O}}_{V A}$ are dimension six operators with $\Delta s=2$, we can restrict ourselves to dimension six operators for the construction of the lattice operators corresponding to them. The set of lattice bare operators with even parity is given by

$$
\begin{aligned}
V V & =\left(\bar{s} \gamma_{\mu} d\right)\left(\bar{s} \gamma_{\mu} d\right), \\
A A & =\left(\bar{s} \gamma_{\mu} \gamma_{5} d\right)\left(\bar{s} \gamma_{\mu} \gamma_{5} d\right), \\
S S & =(\bar{s} d)(\bar{s} d), \\
P P & =\left(\bar{s} \gamma_{5} d\right)\left(\bar{s} \gamma_{5} d\right), \\
T T & =\frac{1}{2}\left(\bar{s} \sigma_{\mu \nu} d\right)\left(\bar{s} \sigma_{\mu \nu} d\right),
\end{aligned}
$$

and the set with odd parity is

$$
\begin{aligned}
V A & =\left(\bar{s} \gamma_{\mu} d\right)\left(\bar{s} \gamma_{\mu} \gamma_{5} d\right), \\
S P & =(\bar{s} d)\left(\bar{s} \gamma_{5} d\right), \\
T \tilde{T} & =\frac{1}{2}\left(\bar{s} \sigma_{\mu \nu} d\right)\left(\bar{s} \sigma_{\mu \nu} \gamma_{5} d\right),
\end{aligned}
$$

where $\sigma_{\mu \nu}=\left[\gamma_{\mu}, \gamma_{\nu}\right] / 2$. We rearrange these operators into the Fierz eigenbasis, which we find convenient when taking fermion contractions for evaluating the Green functions in Eq. (18):

$$
\begin{aligned}
& \mathcal{O}_{0}=V V+A A(+,+), \\
& \mathcal{O}_{1}=S S+T T+P P(+,+), \\
& \mathcal{O}_{2}=S S-\frac{1}{3} T T+P P(-,+), \\
& \mathcal{O}_{3}=(V V-A A)+2(S S-P P)(-,+), \\
& \mathcal{O}_{4}=(V V-A A)-2(S S-P P)(+,+), \\
& \mathcal{O}_{5}=V A(+,+), \\
& \mathcal{O}_{6}=S P+\frac{1}{2} T \tilde{T}(+,-), \\
& \mathcal{O}_{7}=S P-\frac{1}{6} T \tilde{T}(-,-) .
\end{aligned}
$$

Here the first sign after each equation denotes the Fierz eigenvalue and the second the CPS [3] eigenvalue. The Fierz eigenbasis we employ is different from that chosen by the Rome group [7] based on one-loop perturbation theory.

The parity odd operators $\mathcal{O}_{6,7}$ are $C P S$ odd while $\mathcal{O}_{5}$ is $C P S$ even, and hence $\mathcal{O}_{5}$ does not mix with $\mathcal{O}_{6,7}$ under renormalization, where we assume $m_{d}=m_{s}$ in the quark action. Therefore the mixing structure of these operators is given by

$$
\begin{aligned}
\frac{\hat{\mathcal{O}}_{V V+A A}}{Z_{V V+A A}} & =\mathcal{O}_{V V+A A}=z_{0} \mathcal{O}_{0}+z_{1} \mathcal{O}_{1}+\cdots+z_{4} \mathcal{O}_{4}, \\
\frac{\hat{\mathcal{O}}_{V A}}{Z_{V A}} & =\mathcal{O}_{V A}=z_{5} \mathcal{O}_{5},
\end{aligned}
$$

where $Z_{V V+A A}$ and $Z_{V A}$ are overall renormalization factors, and we take $z_{0}=1$.

Let us consider an external state consisting of two $s$ quarks and two $d$ quarks, all having an equal momentum $p$. Under $\lambda^{3}$ chiral rotation the Ward identity (18) for such an external state takes the following form:

$$
\begin{aligned}
F_{V V+A A} \equiv & -2 \rho_{m} Z_{A}^{\mathrm{ext}} \\
& \times \sum_{x}\left\langle P^{a}(x) \frac{1}{2} \hat{O}_{V V+A A}(0) \tilde{s}(p) \tilde{s}(p) \tilde{\tilde{d}}(p) \tilde{\tilde{d}}(p)\right\rangle \\
& -\left\langle\hat{\mathcal{O}}_{V A}(0) \tilde{s}(p) \tilde{s}(p) \tilde{\tilde{d}}(p) \tilde{\tilde{d}}(p)\right\rangle \\
& -\left\langle\frac{1}{2} \hat{O}_{V V+A A}(0) \tilde{s}(p) \tilde{s}(p)\left[\tilde{\bar{d}}(p) \frac{\gamma_{5}}{2}\right] \tilde{\tilde{d}}(p)\right\rangle \\
& -\left\langle\frac{1}{2} \hat{O}_{V V+A A}(0) \tilde{s}(p) \tilde{s}(p) \tilde{\bar{d}}(p)\left[\tilde{\tilde{d}}(p) \frac{\gamma_{5}}{2}\right]\right\rangle \\
& +O(a)=0, \\
F_{V A} \equiv & -2 \rho_{m} Z_{A}^{\mathrm{ext}} \sum_{x}\left\langle P{ }^{a}(x) \hat{O_{V A}}(0) \tilde{s}(p) \tilde{s}(p) \tilde{\bar{d}}(p) \tilde{\tilde{d}}(p)\right\rangle \\
& -\left\langle\frac{1}{2} \hat{O}_{V V+A A}(0) \tilde{s}(p) \tilde{s}(p) \tilde{\tilde{d}}(p) \tilde{\tilde{d}}(p)\right\rangle \\
& -\left\langle\hat{O}_{V A}(0) \tilde{s}(p) \tilde{s}(p)\left[\tilde{\tilde{d}}(p) \frac{\gamma_{5}}{2}\right] \tilde{\tilde{d}}(p)\right\rangle \\
& -\left\langle\hat{O}_{V A}(0) \tilde{s}(p) \tilde{s}(p) \tilde{\bar{d}}(p)\left[\tilde{\tilde{d}}(p) \frac{\gamma_{5}}{2}\right]\right\rangle+O(a)=0 .
\end{aligned}
$$

We obtain the amputated Green functions for $F_{V V+A A}$ and $F_{V A}$ by truncating the external quark propagators according to

$$
\begin{gathered}
\Gamma_{V V+A A} \equiv G_{s}^{-1}(p) G_{s}^{-1}(p) F_{V V+A A} G_{\bar{d}}^{-1}(p) G_{\bar{d}}^{-1}(p), \\
\Gamma_{V A} \equiv G_{s}^{-1}(p) G_{s}^{-1}(p) F_{V A} G_{\bar{d}}^{-1}(p) G_{\bar{d}}^{-1}(p),
\end{gathered}
$$

where $G_{q}^{-1}$ denotes the inverse quark propagator with the flavor $q$. 
Let $P_{i}(i=0, \ldots, 7)$ be the tree-level Dirac components corresponding to the four-quark operators in the Fierz eigenbasis $\mathcal{O}_{i}(i=0, \ldots, 7)$ : e.g.,

$$
P_{0}^{\alpha \beta \delta \lambda}=\gamma_{\mu}^{\alpha \beta} \gamma_{\mu}^{\delta \lambda}+\left(\gamma_{\mu} \gamma_{5}\right)^{\alpha \beta}\left(\gamma_{\mu} \gamma_{5}\right)^{\delta \lambda}
$$

Since QCD conserves parity one can write

$$
\begin{aligned}
\frac{\Gamma_{V V+A A}}{Z_{V V+A A}} & =\Gamma_{5} P_{5}, \\
\frac{\Gamma_{V A}}{Z_{V A}} & =\Gamma_{0} P_{0}+\Gamma_{1} P_{1}+\cdots+\Gamma_{4} P_{4},
\end{aligned}
$$

where the $c$-number coefficients $\Gamma_{0}, \ldots, \Gamma_{5}$ are obtained by applying the suitable projection operators to $\Gamma_{V V+A A} /$ $Z_{V V+A A}$ and $\Gamma_{V A} / Z_{V A}$, e.g.,

$$
\bar{P}_{0}^{\beta \alpha \lambda \delta}=\frac{1}{128}\left[\gamma_{\nu}^{\beta \alpha} \gamma_{\nu}^{\lambda \delta}+\left(\gamma_{5} \gamma_{\nu}\right)^{\beta \alpha}\left(\gamma_{5} \gamma_{\nu}\right)^{\lambda \delta}\right]
$$

corresponding to $P_{0}^{\alpha \beta \delta \lambda}$. Expressing $\hat{\mathcal{O}}_{V V+A A, V A}$ in Eq. (18) in terms of lattice operators, we obtain six equations for the five coefficients $z_{1}, \ldots, z_{5}$ :

$$
\Gamma_{i}=c_{0}^{i}+c_{1}^{i} z_{1}+\cdots+c_{5}^{i} z_{5}=O(a), \quad i=0, \ldots, 5 .
$$

This gives an overconstrained set of equations, and we may choose any five equations to exactly vanish to solve for $z_{i}$ : the remaining equation should automatically be satisfied to $O(a)$. We choose four equations to be those for $i$ $=1, \ldots, 4$, since $\mathcal{O}_{1}, \ldots, \mathcal{O}_{4}$ do not appear in the continuum. The choice of the fifth equation, $i=0$ or 5 , is more arbitrary. We have checked that either $\Gamma_{0}=0$ or $\Gamma_{5}=0$ leads to a consistent result to $O(a)$ for $z_{1}, \ldots, z_{4}$ in the region $p a \lesssim 1$. In the present analysis we choose $\Gamma_{5}=0$.

Let us remark here that the equations obtained in the NPR method [7] corresponds to $\Gamma_{i}=0$ for $i=1, \ldots, 4$ in which the contributions of the first term due to the quark mass contributions and the third term representing chiral rotation of quark fields in the Ward identity (18) are dropped. The authors of Refs. [6,7,11] argued that the NPR method is equivalent to the Ward identities in the limit of large external quark momentum $p$. The reasoning is that the first term is suppressed by one power of $p$ due to the explicit quark mass factor, and the third term does not yield chiral-breaking components since the inverse quark propagator for large $p$ has the form $G^{-1} \propto i \Sigma_{\mu} \gamma_{\mu} p_{\mu}$ which anticommutes with $\gamma_{5}$. Under these circumstances the first, third, and fourth terms of $F_{V A}$ in Eq. (45) become irrelevant for the determination of the mixing coefficients $z_{1}, \ldots, z_{4}$. However, the latter point is not correct at finite lattice spacing. The inverse quark propagator does not anticommute with $\gamma_{5}$ in the large momentum region because the contribution of the Wilson term in the quark propagator becomes larger, and hence not negligible, as the momentum increases. Therefore the third and fourth terms of $F_{V A}$ in Eq. (45) yield components having Dirac structures other than $V V+A A$ after truncating the external quark propagators. In conclusion the NPR method is a part of the Ward identities; at the large external quark momentum $p$ the former becomes equivalent to the latter up to $O(p a)$. In Sec. IV we show the difference between them numerically.

\section{B. Matching of lattice and continuum operators}

In our earlier report [9] we employed the NPR method of Ref. [6] to evaluate the overall renormalization factor $Z_{V V+A A}$ in Eq. (42). For the reasons discussed in Sec. V we use the perturbative estimate in the final analysis presented in this article.

The one-loop perturbative renormalization of the $\Delta_{s}=2$ operator is written in the following way [12]:

$$
\hat{\mathcal{O}}_{V V+A A}=Z_{V V+A A} \mathcal{O}_{0}+\frac{\alpha_{s}}{4 \pi} Z^{*}\left(\frac{1}{3} \mathcal{O}_{1}-\frac{1}{2} \mathcal{O}_{3}-\frac{5}{12} \mathcal{O}_{4}\right),
$$

with $\mathcal{O}_{i}(i=0, \ldots, 4)$ being the Fierz eigenoperators defined in Eqs. (34)-(38). Employing the modified minimal subtraction $(\overline{\mathrm{MS}})$ scheme with naive dimensional regularization (NDR) for the continuum theory the renormalization factors are given by $[12,5]$

$$
\begin{aligned}
Z_{V V+A A} & =1+\frac{\alpha_{s}}{4 \pi}\left[-4 \ln (\mu a)+\Delta_{V V+A A}\right], \\
\Delta_{V V+A A} & =-50.841 \text { for } \mathrm{NDR}, \\
Z^{*} & =9.6431,
\end{aligned}
$$

where $\mu$ is the renormalization scale. The diagonal part $\Delta_{V V+A A}$ is affected by the renormalization scheme in the continuum, while the mixing part $Z^{*}$ is independent. We collect the value of $\Delta_{V V+A A}$ for the dimensional reduction (DRED) scheme in the Appendix.

Including the normalization of quark fields $\sqrt{8 K_{c}} \sqrt{1 / 2 K-3 / 8 K_{c}}[13,14]$ tadpole improved by the factor $u_{0}=\left(8 K_{c}\right)^{-1}$ leads to

$$
\begin{aligned}
Z_{V V+A A}= & \left(\frac{1}{2 K}-\frac{3}{8 K_{c}}\right)^{2} \\
& \times\left[1+\frac{\alpha_{s}}{4 \pi}\left[-4 \ln (\mu a)+\Delta_{V V+A A}+2 \pi \times 5.457\right]\right] .
\end{aligned}
$$

Here $K_{c}$ is the critical hopping parameter where the pion mass vanishes. We use

$$
\frac{1}{8 K_{c}}=1-5.457 \alpha_{s} / 4
$$

in Ref. [15] for the perturbative estimate of $K_{c}$.

With the use of $Z_{V V+A A}^{\mathrm{NDR}}$ we convert the matrix element on the lattice into that in the continuum NDR scheme renormalized at the scale $\mu=1 / a \mathrm{GeV}[16,5]$ : 


$$
B_{K}(\mathrm{NDR}, 1 / a)=\frac{Z_{V V+A A}^{\mathrm{NDR}}}{Z_{A}^{\mathrm{NDR}}} \frac{\left\langle\bar{K}^{0}\left|\mathcal{O}_{V V+A A}\right| K^{0}\right\rangle}{(8 / 3)\left|\left\langle 0\left|A_{\mu}\right| K^{0}\right\rangle\right|^{2}},
$$

where $\mathcal{O}_{V V+A A}$ is defined in Eq. (42), and $Z_{A}$ is the renormalization factor for the axial vector current $A_{\mu}=\bar{s} \gamma_{\mu} \gamma_{5} d$, which is expressed as $[17,15]$

$$
\begin{aligned}
& Z_{A}=1+\frac{\alpha_{s}}{4 \pi} \Delta_{A}, \\
& \Delta_{A}=-21.061 \text { for NDR. }
\end{aligned}
$$

The value of $\Delta_{A}$ for the DRED scheme is given in the Appendix. With the tadpole improvement the expression (60) becomes

$$
Z_{A}=\left(\frac{1}{2 K}-\frac{3}{8 K_{c}}\right)\left[1+\frac{\alpha_{s}}{4 \pi}\left(\Delta_{A}+\pi \times 5.457\right)\right] .
$$

The continuum value at a physical scale $\mu=2 \mathrm{GeV}$ is obtained via a two-loop renormalization group running from $\mu=1 / a \mathrm{GeV}$ :

$$
\begin{aligned}
B_{K}(\mathrm{NDR}, \mu)= & \left(\frac{\alpha_{\overline{\mathrm{MS}}}(\mu)}{\alpha_{\overline{\mathrm{MS}}}(1 / a)}\right)^{\gamma^{(0)} / 2 \beta_{0}} \\
& \times\left[1+\frac{\alpha_{\overline{\mathrm{MS}}}(\mu)-\alpha_{\overline{\mathrm{MS}}}(1 / a)}{4 \pi}\right. \\
& \left.\times\left(\frac{\gamma^{(1)} \beta_{0}-\gamma^{(0)} \beta_{1}}{2 \beta_{0}^{2}}\right)\right] B_{K}(\mathrm{NDR}, 1 / a),
\end{aligned}
$$

where $\beta_{0,1}$ are the leading and next-to-leading coefficients of the $\beta$ function and $\gamma^{(0,1)}$ are those of the anomalous dimension for $\hat{\mathcal{O}}_{V V+A A}$. We take $\beta_{0}=11, \beta_{1}=102, \gamma^{(0)}=4$, and $\gamma^{(1)}=-7$ [18] appropriate for the zero-flavor case corresponding to our quenched calculation of $B_{K}$.

We define another $B$ parameter to investigate the chiral property of the operator $\hat{\mathcal{O}}_{V V+A A}$ :

$$
B_{K}^{P}(\mathrm{NDR}, 1 / a)=\frac{Z_{V V+A A}^{\mathrm{NDR}}}{Z_{P}^{\mathrm{NDR}}} \frac{\left\langle\bar{K}^{0}\left|\mathcal{O}_{V V+A A}\right| K^{0}\right\rangle}{(8 / 3)\left|\left\langle 0|P| K^{0}\right\rangle\right|^{2}}
$$

with $Z_{P}$ the renormalization factor for the pseudoscalar density $P=\bar{s} \gamma_{5} d$. The continuum value of $B_{K}^{P}$ at $2 \mathrm{GeV}$ is obtained by running from $\mu=1 / a$ to $2 \mathrm{GeV}$ according to the two-loop renormalization group. We use $\gamma_{P}^{(0)}=-8$ and $\gamma_{P}^{(1)}=-404 / 3$ [18] for the leading and next-to-leading coefficients of the anomalous dimension of the pseudoscalar density in the zero-flavor case. The one-loop perturbative expression for $Z_{P}$ with the tadpole improvement is given by $[17,15]$

$$
Z_{P}=\left(\frac{1}{2 K}-\frac{3}{8 K_{c}}\right)\left[1+\frac{\alpha_{s}}{4 \pi}\left[8 \ln (\mu a)+\Delta_{P}+\pi \times 5.457\right]\right],
$$

$$
\Delta_{P}=-30.128 \text { for NDR. }
$$

The value of $\Delta_{P}$ for the DRED scheme is given in the Appendix.

The overall renormalization factors $Z_{V V+A A}, Z_{A}$, and $Z_{P}$ can be alternatively determined by the NPR method [6]. The NPR method closely follows what is usually done in the perturbative renormalization. The vertex corrections are extracted from the amputated Green functions for off-shell external quark states with momentum $p$ in the Landau gauge according to

$$
\begin{gathered}
G_{s}^{-1}(p) G_{s}^{-1}(p)\left\langle\mathcal{O}_{V V+A A}(0) \tilde{s}(p) \tilde{s}(p) \tilde{\bar{d}}(p) \tilde{\bar{d}}(p)\right\rangle \\
\times G_{\bar{d}}^{-1}(p) G_{\bar{d}}^{-1}(p)=\Lambda_{0}(p) P_{0}+\cdots, \\
G_{s}^{-1}(p)\left\langle A_{\mu}(0) \tilde{s}(p) \tilde{\tilde{d}}(p)\right\rangle G_{\bar{d}}^{-1}(p)=\Lambda_{\gamma_{\mu} \gamma_{5}}(p) P_{\gamma_{\mu} \gamma_{5}}+\cdots, \\
G_{s}^{-1}(p)\langle P(0) \tilde{s}(p) \tilde{\bar{d}}(p)\rangle G_{\bar{d}}^{-1}(p)=\Lambda_{\gamma_{5}}(p) P_{\gamma_{5}}+\cdots,
\end{gathered}
$$

where $P$ 's are the tree-level Dirac components with $P_{0}$ given in Eq. (48) and $P_{\gamma_{\mu} \gamma_{5}, \gamma_{5}}$ defined by

$$
\begin{gathered}
P_{\gamma_{\mu} \gamma_{5}}^{\alpha \beta}=\left(\gamma_{\mu} \gamma_{5}\right)^{\alpha \beta}, \\
P_{\gamma_{5}}^{\alpha \beta}=\gamma_{5}{ }^{\alpha \beta} .
\end{gathered}
$$

We should note that the amputated Green functions for the bilinear operators can have extra Dirac components besides their tree-level ones, which originate from contribution of the higher dimensional operators. The quark wave-function renormalization factor $Z_{q}(p)$ is extracted from the quark self-energy

$$
Z_{q}(p)=\frac{\operatorname{Tr}\left[-i \Sigma_{\mu} \gamma_{\mu} \sin \left(p_{\mu}\right) G_{q}^{-1}(p)\right]}{12 \Sigma_{\mu} \sin ^{2}\left(p_{\mu}\right)},
$$

where the trace is applied for the Dirac and color indices. In terms of the vertex corrections and the wave-function renormalization factor one calculates $Z_{V V+A A}, Z_{A}$, and $Z_{P}$ imposing the following conditions:

$$
\begin{gathered}
Z_{V V+A A}(p) Z_{q}^{-2}(p) \Lambda_{0}(p)=1, \\
Z_{A}(p) Z_{q}^{-1}(p)\left[\frac{1}{4} \sum_{\mu} \Lambda_{\gamma_{\mu} \gamma_{5}}(p)\right]=1 \\
Z_{P}(p) Z_{q}^{-1}(p) \Lambda_{\gamma_{5}}(p)=1 .
\end{gathered}
$$

This renormalization scheme is called the regularization independent (RI) scheme. In this scheme the renormalization constants depend on the external state and the gauge. The perturbative values of the renormalization constants $\Delta_{V V+A A}, \Delta_{A}$, and $\Delta_{P}$ defined in Eqs. (54), (60), and (65) for the RI scheme are given in the Appendix. 
TABLE I. Parameters of our simulations. See text for details.

\begin{tabular}{lcccc}
\hline \hline$\beta$ & 5.9 & 6.1 & 6.3 & 6.5 \\
\hline$L^{3} \times T$ & $24^{3} \times 64$ & $32^{3} \times 64$ & $40^{3} \times 96$ & $48^{3} \times 96$ \\
No. conf. & 300 & 100 & 50 & 24 \\
Thermalization & 22000 & 32000 & 45000 & 72000 \\
Interval & 2000 & 2000 & 5000 & 8000 \\
$K$ & 0.15862 & 0.15428 & 0.15131 & 0.14925 \\
& 0.15785 & 0.15381 & 0.15098 & 0.14901 \\
& 0.15708 & 0.15333 & 0.15066 & 0.14877 \\
& 0.15632 & 0.15287 & 0.15034 & 0.14853 \\
$K_{c}$ & $0.15986(3)$ & $0.15502(2)$ & $0.15182(2)$ & $0.14946(3)$ \\
$m_{s} a / 2=m_{d} a / 2$ & $0.0294(14)$ & $0.0198(16)$ & $0.0144(17)$ & $0.0107(16)$ \\
$a^{-1}[\mathrm{GeV}]$ & $1.95(5)$ & $2.65(11)$ & $3.41(20)$ & $4.30(29)$ \\
$L a[\mathrm{fm}]$ & 2.4 & 2.4 & 2.3 & 2.2 \\
$\left\langle\right.$ Tr $\left.U_{P}\right\rangle$ & 0.582 & 0.604 & 0.622 & 0.638 \\
$\alpha_{\overline{\mathrm{MS}}}(1 / a)$ & 0.1922 & 0.1739 & 0.1596 & 0.1480 \\
$\delta_{p^{2}}{ }^{(*)^{2}} a^{2}$ & 1.11 & 1.11 & 1.15 & 1.12 \\
Fitting range for $m_{\pi}, m_{\rho}, \rho_{m}, Z_{A}^{\mathrm{ext}}$ & 0.9595 & 0.5012 & 0.2988 & 0.2056 \\
Fitting range for $B_{K}, B_{K}^{P}$ & $12-20$ & $14-24$ & $17-27$ & $20-30$ \\
\hline \hline
\end{tabular}

\section{DETAILS OF NUMERICAL SIMULATION}

\section{A. Data sets}

Our calculations are made with the Wilson quark action and the plaquette gauge action at $\beta=5.9-6.5$ in quenched QCD. Table I summarizes our run parameters. Gauge configurations are generated with the five-hit pseudo-heat-bath algorithm. At each value of $\beta$ four values of the hopping parameter $K$ are adopted such that the physical point for the $K$ meson can be interpolated. The critical hopping parameter $K_{c}$ is determined by extrapolating results for $m_{\pi}^{2}$ at the four hopping parameters linearly in $1 / 2 K$ to $m_{\pi}^{2}=0$. We take the down and strange quarks to be degenerate. The value of half the strange quark mass $m_{s} a / 2$ is then estimated from the experimental ratio $m_{K} / m_{\rho}=0.648$.

The inverse lattice spacing $a^{-1}$ is determined from the $\rho$ meson mass $m_{\rho}=770 \mathrm{MeV}$. The physical size of lattice is chosen to be approximately constant at $L a \approx 2.4 \mathrm{fm}$. To calculate the perturbative renormalization factors, we employ the strong coupling constant at the scale $1 / a$ in the $\overline{\mathrm{MS}}$ scheme, evaluated by a two-loop renormalization group running starting from $1 / g_{\overline{\mathrm{MS}}}^{2}(\pi / a)=\left\langle\operatorname{Tr} U_{P}\right\rangle / g_{\text {latt }}^{2}+0.0246$ with $\left\langle\operatorname{Tr} U_{P}\right\rangle$ the averaged value of the plaquette.

In order to calculate the mixing coefficients $z_{i}$ ( $i$ $=1, \ldots, 5)$ with the Ward identity method and the renormalization factors $Z_{V V+A A}, Z_{A}$, and $Z_{P}$ with the NPR method, the latter for purpose of comparison with the perturbative values, we prepare a set of external quark momenta $p^{(i)}$ $=\left(p_{x}^{(i)}, p_{y}^{(i)}, p_{z}^{(i)}, p_{t}^{(i)}\right) \quad(i=1 \cdots \sim 40)$. These momenta are chosen recursively according to the condition that the $i$ $+1)$ th momentum $p^{(i+1)} a$ is the minimum number satisfying

$$
\left(p^{(i+1)} a\right)^{2} \geqslant \delta_{p^{2}}\left(p^{(i)} a\right)^{2},
$$

$$
p_{x}^{(i+1)} \leqslant p_{y}^{(i+1)} \leqslant p_{z}^{(i+1)},
$$

for a given value of the increment parameter $\delta_{p^{2}}$ starting with $p^{(1)} a=(0,0,0,2 \pi / T)$ where $T$ denotes the temporal lattice size. In the case of multiple choices for the $i+$ first momentum we take the momentum that has the largest value of $p_{t}^{(i+1)}$. The choice of the value of $\delta_{p^{2}}$ is listed in Table I. We employ the momentum having $p^{(*)} \approx 2 \mathrm{GeV}$ among the $p^{(i)}$ 's for the analysis of $B$ parameters. We estimate errors by the single elimination jackknife procedure for all measured quantities except for the extrapolation to the continuum limit as a function of $a$.

\section{B. Calculational procedure}

Our calculations are carried out in three steps. We first calculate $m_{\pi}, m_{\rho}, \rho_{m}$, and $Z_{A}^{\text {ext }}$ using the hadron Green functions. For this purpose quark propagators are solved in the Landau gauge for the point source located at the origin with the periodic boundary condition imposed in all four directions. Following Eq. (19) we can extract the $\rho_{m}$ parameter from the ratio

$$
\begin{aligned}
\rho_{m} & =\frac{1}{2}\left[\frac{\sum_{\vec{x}}\left\langle\nabla_{4} A_{4}^{\mathrm{ext}, 3}(\vec{x}, t) P^{3}(\overrightarrow{0}, 0)\right\rangle}{2 \Sigma_{\vec{x}}\left\langle P^{3}(\vec{x}, t) P^{3}(\overrightarrow{0}, 0)\right\rangle}+(t \rightarrow T-t+1)\right] \\
& \rightarrow \frac{\left\langle 0\left|\nabla_{4} A_{4}^{\mathrm{ext}, 3}\right| \pi^{3}\right\rangle}{2\left\langle 0\left|P^{3}\right| \pi^{3}\right\rangle}, \quad 0 \ll t \ll T-1
\end{aligned}
$$

by fitting a plateau as a function of $t$, where

$$
\nabla_{4} A_{4}^{\mathrm{ext}, 3}(\vec{x}, t)=A_{4}^{\mathrm{ext}, 3}(\vec{x}, t)-A_{4}^{\mathrm{ext}, 3}(\vec{x}, t-1),
$$


TABLE II. Meson masses, $\rho_{m}$ parameter, and renormalization factor for the extended axial vector current at $\beta=5.9-6.5$ in quenched QCD.

\begin{tabular}{|c|c|c|c|c|c|c|}
\hline$\beta$ & $K$ & $m_{\pi}$ & $m_{\rho}$ & $m_{\pi} / m_{\rho}$ & $\rho_{m}$ & $Z_{A}^{\mathrm{ext}}$ \\
\hline \multirow[t]{4}{*}{5.9} & 0.15862 & $0.2346(19)$ & $0.443(10)$ & $0.530(12)$ & $0.03307(33)$ & $1.328(58)$ \\
\hline & 0.15785 & $0.2980(15)$ & $0.4636(49)$ & $0.6427(67)$ & $0.05433(32)$ & $0.941(19)$ \\
\hline & 0.15708 & $0.3513(12)$ & $0.4897(33)$ & $0.7172(45)$ & $0.07617(31)$ & $0.925(13)$ \\
\hline & 0.15632 & $0.3982(11)$ & $0.5181(25)$ & $0.7687(33)$ & $0.09836(29)$ & $0.919(10)$ \\
\hline \multirow[t]{4}{*}{6.1} & 0.15428 & $0.1677(16)$ & $0.323(12)$ & $0.520(19)$ & $0.02239(29)$ & $0.970(36)$ \\
\hline & 0.15381 & $0.2135(16)$ & $0.3467(60)$ & $0.616(11)$ & $0.03732(29)$ & $0.935(23)$ \\
\hline & 0.15333 & $0.2527(15)$ & $0.3688(40)$ & $0.6853(81)$ & $0.05276(29)$ & $0.933(17)$ \\
\hline & 0.15287 & $0.2864(14)$ & $0.3892(31)$ & $0.7358(63)$ & $0.06778(29)$ & $0.933(14)$ \\
\hline \multirow[t]{4}{*}{6.3} & 0.15131 & $0.1282(23)$ & $0.254(14)$ & $0.504(27)$ & $0.01725(34)$ & $0.981(68)$ \\
\hline & 0.15098 & $0.1641(20)$ & $0.2643(65)$ & $0.621(15)$ & $0.02889(29)$ & $0.949(41)$ \\
\hline & 0.15066 & $0.1933(18)$ & $0.2797(44)$ & $0.691(11)$ & $0.04024(26)$ & $0.928(32)$ \\
\hline & 0.15034 & $0.2195(17)$ & $0.2960(35)$ & $0.7413(88)$ & $0.05169(24)$ & $0.916(28)$ \\
\hline \multirow[t]{4}{*}{6.5} & 0.14925 & $0.0782(39)$ & $0.189(13)$ & $0.414(31)$ & $0.00860(42)$ & $0.95(14)$ \\
\hline & 0.14901 & $0.1119(32)$ & $0.2079(79)$ & $0.538(21)$ & $0.01759(42)$ & $0.951(66)$ \\
\hline & 0.14877 & $0.1394(29)$ & $0.2232(60)$ & $0.625(18)$ & $0.02658(41)$ & $0.938(47)$ \\
\hline & 0.14853 & $0.1632(25)$ & $0.2368(45)$ & $0.689(14)$ & $0.03565(39)$ & $0.929(37)$ \\
\hline
\end{tabular}

$$
\begin{aligned}
A_{4}^{\mathrm{ext}, 3}(\vec{x}, t)= & \frac{1}{4}\left[\bar{u}(\vec{x}, t) \gamma_{4} \gamma_{5} U_{4}(\vec{x}, t) u(\vec{x}, t+1)\right. \\
& \left.+\bar{u}(\vec{x}, t+1) \gamma_{4} \gamma_{5} U_{4}^{\dagger}(\vec{x}, t) u(\vec{x}, t)-(u \leftrightarrow d)\right],
\end{aligned}
$$

$$
P^{3}(\vec{x}, t)=\frac{1}{2}\left[\bar{u}(\vec{x}, t) \gamma_{5} u(\vec{x}, t)-(u \leftrightarrow d)\right]
$$

In order to determine $Z_{A}^{\text {ext }}$ we make a zero-momentum projection in $y$ in Eq. (20):

$$
\begin{gathered}
-2 \rho_{m} Z_{A}^{\text {ext }, 3} \sum_{x, \vec{y}}\left\langle P^{3}(x) A_{\mu}^{+}(\vec{y}, t) V_{\nu}^{-}(\overrightarrow{0}, 0)\right\rangle \\
=\frac{Z_{V}}{Z_{A}} \sum_{\vec{y}}\left\langle V_{\mu}^{+}(\vec{y}, t) V_{\nu}^{-}(\overrightarrow{0}, 0)\right\rangle \\
-\frac{Z_{A}}{Z_{V}} \sum_{\vec{y}}\left\langle A_{\mu}^{+}(\vec{y}, t) A_{\nu}^{-}(\overrightarrow{0}, 0)\right\rangle,
\end{gathered}
$$

where the flavor matrices $\lambda^{+}$and $\lambda^{-}$are defined by

$$
\lambda^{+}=\left(\begin{array}{c}
0,1,0 \\
0,0,0 \\
0,0,0
\end{array}\right), \quad \lambda^{-}=\left(\begin{array}{c}
0,0,0 \\
1,0,0 \\
0,0,0
\end{array}\right)
$$

At each time slice $t$ we obtain $Z_{A}^{\text {ext,3 }}$ and $Z_{A} / Z_{V}$ from the two independent equations corresponding to the choices $\mu=\nu$ $=4$ and $\mu=\nu=i(i=1,2,3)$ in Eq. (82). In Table II we summarize the values of $m_{\pi}, m_{\rho}, \rho_{m}$, and $Z_{A}^{\text {ext }}$ for the four hopping parameters at each $\beta$.

In terms of $\rho_{m}$ and $Z_{A}^{\text {ext }}$ we determine the mixing coefficients $z_{i}(i=1, \ldots, 5)$ according to the Ward identity (18). The quark Green functions having finite space-time momenta are constructed with the point source quark propagators in the Landau gauge. For calculation of the first term in the Ward identity (18), we employ the source method [19] to insert the pseudoscalar density.

The $B_{K}$ parameter is extracted from the following ratio of the hadron-three-point function divided by the two-point functions

$$
R_{A}(t)=\frac{\sum_{\vec{x}, \vec{y}, \vec{z}}\left\langle\mathcal{O}_{\bar{K}^{0}}(\vec{x}, T-1) \hat{\mathcal{O}}_{V V+A A}(\vec{y}, t) \mathcal{O}_{K^{0}}^{\dagger}(\vec{z}, 0)\right\rangle}{(8 / 3) \sum_{\vec{x}, \vec{y}}\left\langle\mathcal{O}_{\bar{K}^{0}}(\vec{x}, T-1) \hat{A}(\vec{y}, t)\right\rangle \Sigma_{\vec{y}^{\prime}, \vec{z}}\left\langle\hat{A}\left(\vec{y}^{\prime}, t\right) \mathcal{O}_{K^{0}}^{\dagger}(\vec{z}, 0)\right\rangle} \rightarrow \frac{1}{L^{3}} B_{K}(\mathrm{NDR}, 1 / a), \quad 0 \ll t \ll T-1,
$$

where operators are defined by

$$
\begin{aligned}
& \mathcal{O}_{K^{0}}(\vec{x}, t)=\bar{s}(\vec{x}, t) \gamma_{5} d(\vec{x}, t), \\
& \mathcal{O}_{\bar{K}^{0}}(\vec{x}, t)=\bar{d}(\vec{x}, t) \gamma_{5} s(\vec{x}, t),
\end{aligned}
$$

$$
\begin{gathered}
\hat{\mathcal{O}}_{V V+A A}(\vec{x}, t)=\sum_{i=0}^{4} Z_{V V+A A} z_{i} \mathcal{O}_{i}(\vec{x}, t), \\
\hat{A}(\vec{x}, t)=Z_{A} \bar{s}(\vec{x}, t) \gamma_{4} \gamma_{5} d(\vec{x}, t),
\end{gathered}
$$


with $Z_{V V+A A}$ and $Z_{A}$ given in Eqs. (57) and (62). The contribution of each operator $\mathcal{O}_{i}(i=0, \ldots, 4)$ to $B_{K}(\mathrm{NDR}, 1 / a)$ can be measured by the ratio

$$
R_{A}^{i}(t)=\frac{\sum_{\vec{x}, \vec{y}, \vec{z}}\left\langle\mathcal{O}_{\bar{K}^{0}}(\vec{x}, T-1) Z_{V V+A A} z_{i} \mathcal{O}_{i}(\vec{y}, t) \mathcal{O}_{K^{0}}^{\dagger}(\vec{z}, 0)\right\rangle}{(8 / 3) \sum_{\vec{x}, \vec{y}}\left\langle\mathcal{O}_{\bar{K}^{0}}(\vec{x}, T-1) \hat{A}(\vec{y}, t)\right\rangle \Sigma_{\vec{y}^{\prime}, \vec{z}}\left\langle\hat{A}\left(\vec{y}^{\prime}, t\right) \mathcal{O}_{K^{0}}^{\dagger}(\vec{z}, 0)\right\rangle} \rightarrow \frac{1}{L^{3}} \frac{\left\langle\bar{K}^{0}\left|Z_{V V+A A} z_{i} \mathcal{O}_{i}\right| K^{0}\right\rangle}{(8 / 3)\left|\left\langle 0|\hat{A}| K^{0}\right\rangle\right|^{2}}, \quad 0 \ll t \ll T-1 .
$$

The sum of $R_{A}^{i}(t)(i=0, \ldots, 4)$ is equal to $R_{A}(t)$. The parameter $B_{K}^{P}(\mathrm{NDR}, 1 / a)$ defined in Eq. (64) is obtained from the ratio

$$
R_{P}(t)=\frac{\sum_{\vec{x}, \vec{y}, \vec{z}}\left\langle\mathcal{O}_{\bar{K}^{0}}(\vec{x}, T-1) \hat{\mathcal{O}}_{V V+A A}(\vec{y}, t) \mathcal{O}_{K^{0}}^{\dagger}(\vec{z}, 0)\right\rangle}{(8 / 3) \sum_{\vec{x}, \vec{y}}\left\langle\mathcal{O}_{\bar{K}^{0}}(\vec{x}, T-1) \hat{P}(\vec{y}, t)\right\rangle \Sigma_{\vec{y}^{\prime}, \vec{z}}\left\langle\hat{P}\left(\vec{y}^{\prime}, t\right) \mathcal{O}_{K^{0}}^{\dagger}(\vec{z}, 0)\right\rangle} \rightarrow \frac{1}{L^{3}} B_{K}^{P}(\mathrm{NDR}, 1 / a), \quad 0 \ll t \ll T-1,
$$

where the renormalized pseudoscalar density is

$$
\hat{P}(\vec{x}, t)=Z_{P} \bar{s}(\vec{x}, t) \gamma_{5} d(\vec{x}, t)
$$

with $Z_{P}$ in Eq. (65). For calculation of the ratios $R_{A}, R_{A}^{i}$, and $R_{P}$ we solve quark propagators without gauge fixing employing wall sources placed at the edges of lattice where the Dirichlet boundary condition is imposed in the time direction.

The value of $B_{K}$ obtained with the Ward identity method depends on the external quark momentum $p^{(i)}$ at which the mixing coefficients are evaluated. To investigate the quark mass dependence and $a$ dependence of $B_{K}$ we employ the averaged value of $B_{K}$ over the five momenta from $p^{(*-2)}$ to $p^{(*+2)}$ where $p^{(*)}$ represents the momentum nearest to 2 $\mathrm{GeV}$. We employ the same procedure for the analysis of $B_{K}^{P}$.

\section{RESULTS FOR MIXING COEFFICIENTS}

In Fig. 1 we plot a typical result for the mixing coefficients $z_{i}(i=1, \ldots, 4)$ as a function of the external quark momenta for the case of $K=0.15034$ at $\beta=6.3$. In order to evaluate the mixing coefficients we need to choose a specific scale $p^{(*)}$ that satisfies the condition $p^{(*)} a \ll 1$ to avoid cutoff contaminations. We observe that the mixing coefficients show only weak dependence over a wide momentum range $0.02 \lesssim p^{2} a^{2} \leqslant 1.0$, albeit $z_{1}$ and $z_{2}$ have large errors in the small momentum region $p^{2} a^{2} \lesssim 0.1$. This enables us to evaluate the mixing coefficients with small uncertainties from the choice of the momentum $p^{(*)}$. We adopt the value $p^{(*)} \approx 2 \mathrm{GeV}$, which we find to always fall within the range of a plateau for our runs at $\beta=5.9-6.5$.

Let us compare the mixing coefficients obtained by the Ward identity (WI) method with those by the NPR. Since the NPR method does not employ the full Ward identity of Eq. (18), it is important to investigate differences in the mixing coefficients between the NPR and the Ward identity methods. In Fig. 2 we present the result for the mixing coefficients $z_{i}$ obtained with the NPR method. The NPR result shows a strong scale dependence in the region $p^{2} a^{2} \lesssim 0.3$, which contrasts to the Ward identity result in Fig. 1. We suspect that this behavior of the NPR result originates from physical nonperturbative contributions, which survive even in the continuum limit (see also Sec. V). Although we ob- serve a similar scale dependence for the two results beyond the scale $p^{2} a^{2} \sim 0.3$, the numerical values for $z_{2}$ and $z_{3}$ show clear deviations beyond the error bars between the WI result and that from the NPR for momenta as large as $p^{2} a^{2} \sim 2$. This is contrary to the expectation that the NPR would be equivalent to the Ward identities in the limit of large external quark momenta $[6,7,11]$.

The difference between the NPR and the WI methods comes from the first and third terms in Eq. (18). To investigate the contribution of each term to the mixing coefficients we reevaluate the mixing coefficients using the Ward identities without the first term or the third term. The former result is plotted in Fig. 3(a) and the latter one in Fig. 3(b). Comparison between Fig. 3(a) and Fig. 1 demonstrates that the contribution of the first term to the mixing coefficients is remarkable in the lower momentum region $p^{2} a^{2} \leq 0.3$. Above this scale, the first term seems to play a minor role on the determination of the mixing coefficients. On the other hand, comparing Fig. 3(b) with Fig. 1 the essential contribution of the third term to the mixing coefficients is observed over a wide range of external momentum, even up to $p^{2} a^{2}$ $\sim 2$. In the Ward identity method we can neglect neither the first term nor the third one.

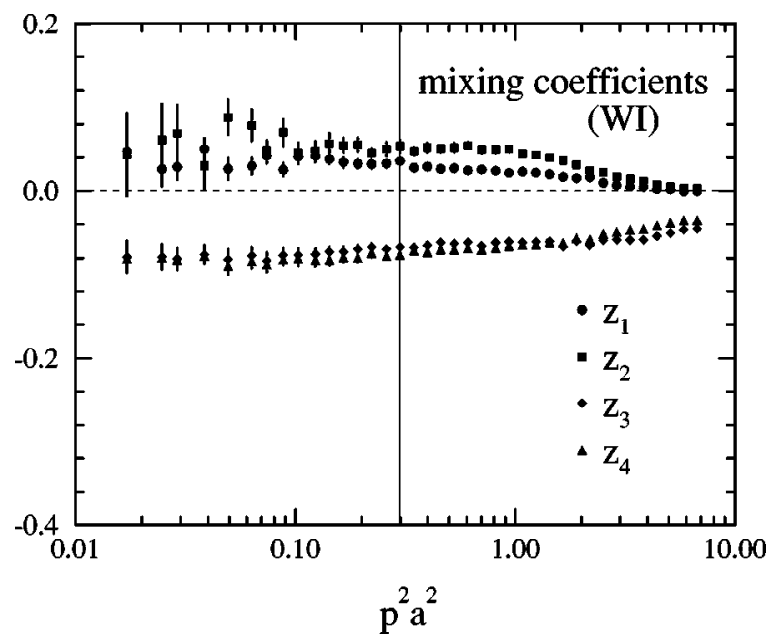

FIG. 1. Mixing coefficients $z_{1}, \ldots, z_{4}$ obtained with the Ward identity method plotted as a function of external momentum squared $p^{2} a^{2}$ for $K=0.15034$ at $\beta=6.3$. Vertical line corresponds to $p^{(*)} \approx 2 \mathrm{GeV}$. 


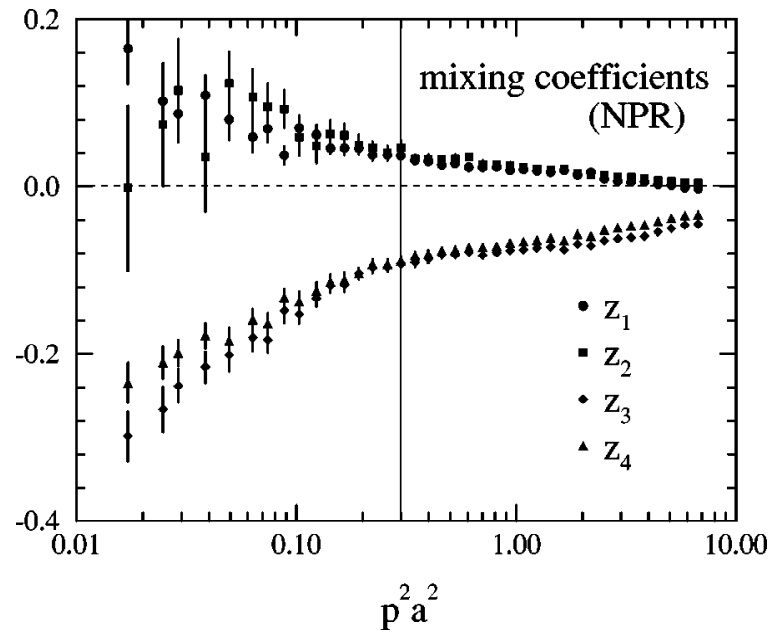

FIG. 2. Mixing coefficients $z_{1}, \ldots, z_{4}$ obtained with the NPR method. Parameters are the same as in Fig. 1.

Figure 4 shows the quark mass dependence of the mixing coefficients $z_{i}(i=1, \ldots, 4)$ evaluated at the scale $p^{(*)}$ (filled symbols) for the case of $\beta=6.3$. For comparison we also plot the perturbative (PT) estimate for $z_{i}$ (open symbols), which are given in Eq. (53), i.e.,

$$
\begin{gathered}
z_{1}=\frac{\alpha_{s}}{4 \pi} Z^{*}\left(+\frac{1}{3}\right), \\
z_{2}=0, \\
z_{3}=\frac{\alpha_{s}}{4 \pi} Z^{*}\left(-\frac{1}{2}\right), \\
z_{4}=\frac{\alpha_{s}}{4 \pi} Z^{*}\left(-\frac{5}{12}\right),
\end{gathered}
$$

where $\alpha \overline{\mathrm{MS}}(1 / a)$ is used for the strong coupling constant. We observe little quark mass dependence for the mixing coefficients.

In Fig. 5 we present the $a$ dependence of the mixing coefficients $z_{i}(i=1, \ldots, 4)$ evaluated at the scale $p^{(*)}$ employing the heaviest quark mass at each $\beta$. We observe that the $a$ dependence of the mixing coefficients determined by the Ward identities is steeper compared to that of the PT estimates to one-loop order. The magnitude of each mixing coefficient for the WI method varies nearly in proportion to $a$, which reduces by $50 \%$ between $m_{\rho} a \approx 0.4$ and $m_{\rho} a \approx 0.2$. A possible source of this $a$ dependence of the mixing coefficients is the $O(a)$ term in Eq. (18): contributions of the $O(a)$ term are absorbed in the mixing coefficients to satisfy the continuum Ward identities at finite lattice spacing.

Comparing the mixing coefficients for the Ward identity method and those of perturbation theory in Figs. 4 and 5, we note that a large value of $z_{2}$ determined by the Ward identities sharply contrasts with the one-loop perturbative result $z_{2}=0$. The magnitude of this discrepancy appears larger than that possibly explained by two-loop contributions; squaring a typical magnitude of one-loop terms in Fig. 5 only yields a
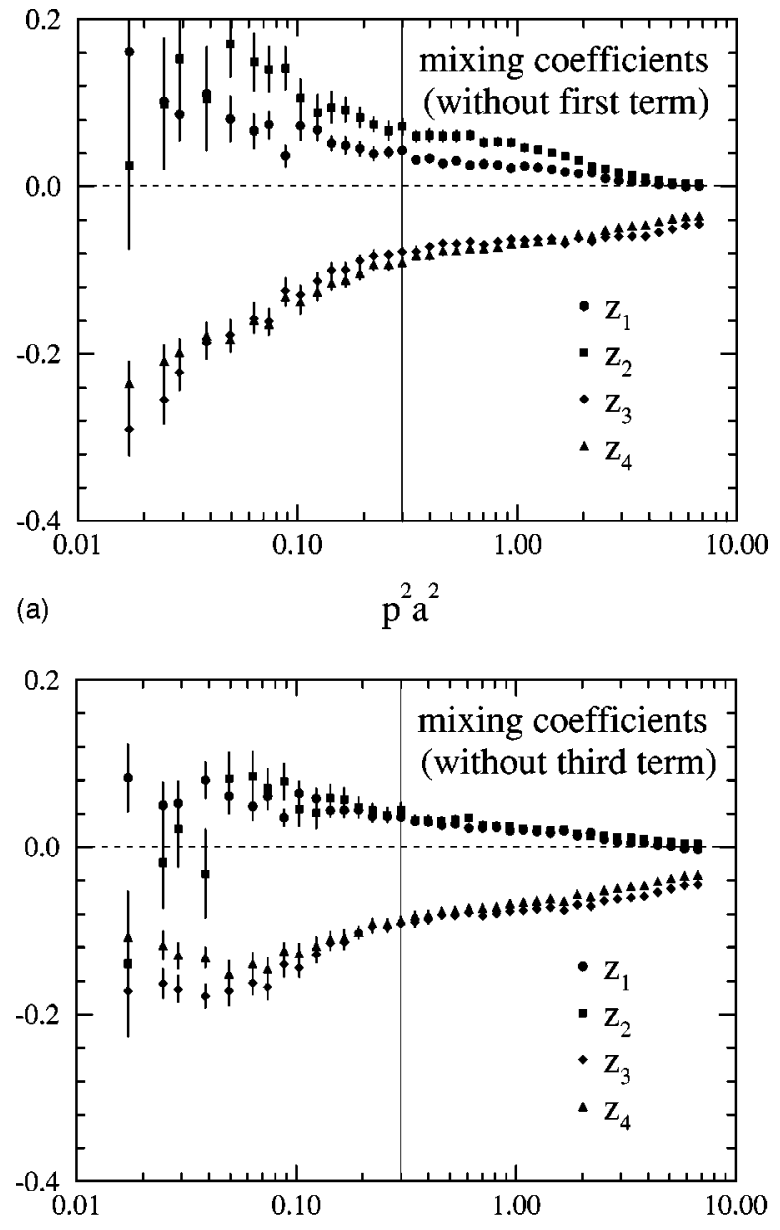

(b)

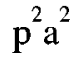

FIG. 3. Mixing coefficients $z_{1}, \ldots, z_{4}$ obtained with the Ward identity (18) neglecting (a) the first term or (b) the third one. Parameters are the same as in Fig. 1.

value of order $\sim 0.001$. Discrepancies also exist for the other coefficients, albeit less conspicuous in that the PT results agree with those of WI in sign and rough orders of magnitude. In particular the magnitude of $z_{4}$ is larger than that for $z_{3}$ for all values of $\beta$, which is contrary to the perturbative result.

For our study of the $B$ parameter the mixing coefficient $z_{5}$ for the parity-odd operator $\hat{\mathcal{O}}_{V A}$ is not directly relevant. However, it is instructive to examine the scale dependence of $z_{5}$, because it would take the value $z_{5}=1$ in the absence of cutoff dependent chiral symmetry breaking effects. In Fig. 6 we plot a typical result for $z_{5}$. We find a scale dependence stronger than those of $z_{i}(i=1, \ldots, 4)$ for parity-even operators toward large momenta; the value of $z_{5}$ significantly deviates from unity as the momentum increases, which measures the magnitude of cutoff effects. The quark mass dependence of $z_{5}$ evaluated at $p^{(*)}$ is shown in Fig. 7. The value of $z_{5}$ slightly increases as the quark mass decreases. We do not consider the strong scale dependence of $z_{5}$ to be particularly alarming since $z_{5}$ evaluated at a fixed physical scale $p^{(*)}$ approaches unity toward the continuum limit as shown in Fig. 8. 


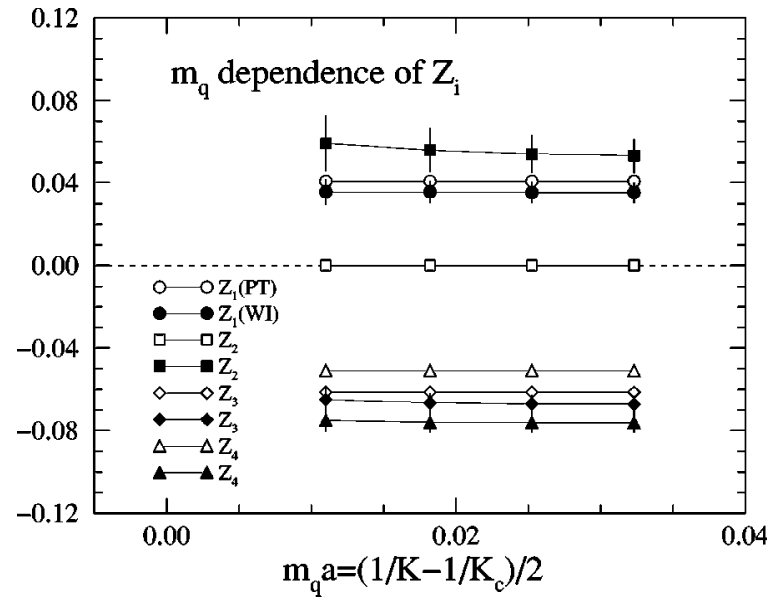

FIG. 4. Quark mass dependence of mixing coefficients $z_{1}, \ldots, z_{4}$ evaluated at $p^{(*)} \approx 2 \mathrm{GeV}$ using the Ward identity (WI, filled symbols) method at $\beta=6.3$. Perturbative (PT, open symbols) results are also plotted for comparison.

\section{RESULTS FOR OVERALL RENORMALIZATION FACTORS WITH THE NPR METHOD}

The NPR method is a possible way to estimate the overall renormalization factors $Z_{V V+A A}, Z_{A}$, and $Z_{P}$. In Fig. 9 we plot $Z_{V V+A A}, Z_{A}$, and $Z_{P}$ in the RI scheme as a function of $p^{2} a^{2}$ for the case of $K=0.15034$ at $\beta=6.3$. For comparison we also draw the tadpole-improved one-loop perturbative estimates (solid lines) in the RI scheme. The NPR result for $Z_{V V+A A}$ in Fig. 9(a) shows an agreement with the perturbative estimate in the region $0.1 \lesssim p^{2} a^{2} \lesssim 0.5$. The dotted curve in Fig. 9(a) represents the $\mathcal{O}_{0}$ contribution to $Z_{V V+A A}$, which is obtained by neglecting contributions of the mixed operators $\mathcal{O}_{i}(i=1, \ldots, 4)$. We observe that the contributions of the mixed operators, leading of which is the two-loop radiative corrections, are quite small.

Figure 9(b) shows that $Z_{A}$ has a strong $p^{2} a^{2}$ dependence below $p^{2} a^{2} \sim 0.3$. This behavior is contrary to the expecta-

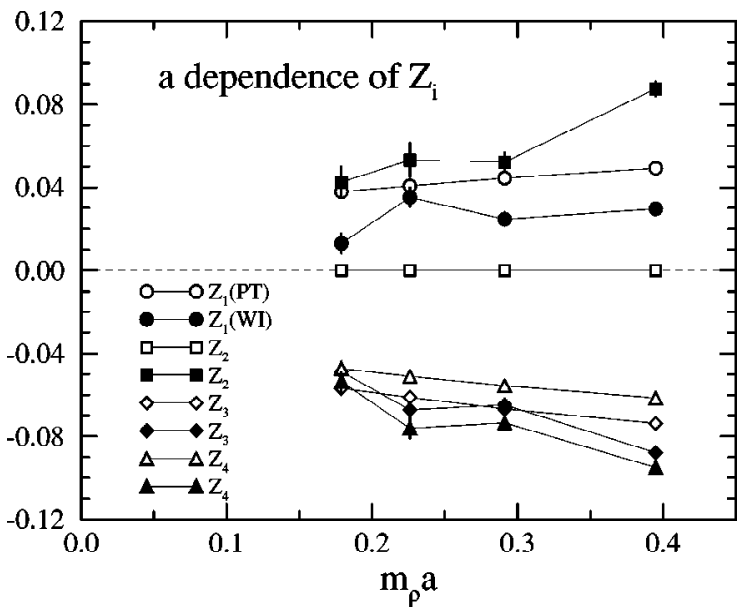

FIG. 5. Comparison of mixing coefficients $z_{1}, \ldots, z_{4}$ evaluated at $p^{(*)} \approx 2 \mathrm{GeV}$ using the Ward identity (WI, filled symbols) method and the perturbative (PT, open symbols) one as a function of $m_{\rho} a$.

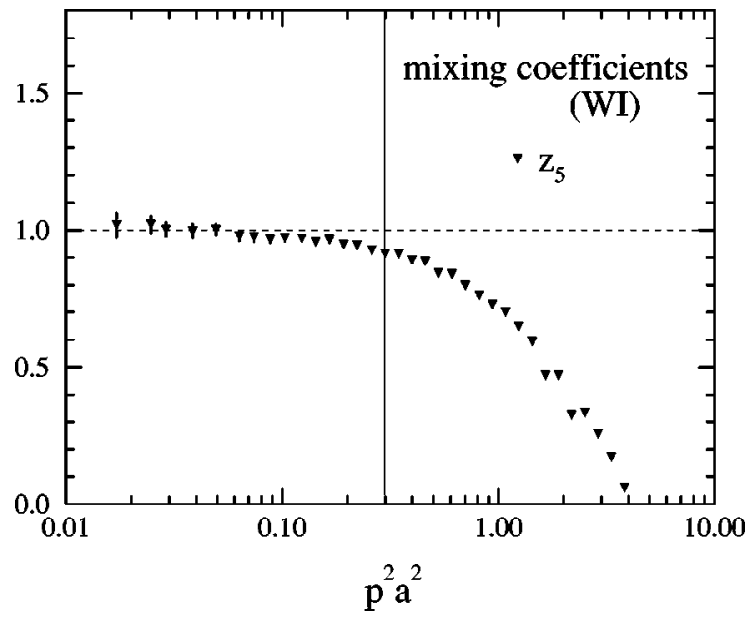

FIG. 6. Same as Fig. 1 for mixing coefficient $z_{5}$.

tion that $Z_{A}$ should be independent of $p^{2} a^{2}$ as the axial vector current has no anomalous dimension. The unexpected behavior may be ascribed to nonperturbative contaminations due to the pion pole which could give an important contribution at low external quark momentum [6]. In Fig. 9(c) we observe a large deviation between the NPR result for $Z_{P}$ and that from perturbation theory below $p^{2} a^{2} \sim 1$. We suspect that this discrepancy is also due to the nonperturbative effects from the pion pole. It should be remarked that the contamination due to the pion pole is not a lattice artifact, and hence survives even after taking the continuum limit.

We note that our NPR results for $Z_{A}$ and $Z_{P}$ are consistent with those of recent studies [20-22]. In particular evidence for the existence of pion pole contribution in $Z_{P}$ has been reported in Ref. [22] for the Kogut-Susskind action and in Ref. [21] for the Wilson case.

For the calculation of $B$ parameters the ratios $Z_{V V+A A} / Z_{A}^{2}$ and $Z_{V V+A A} / Z_{P}^{2}$ are more relevant. We show the scale dependence of $Z_{V V+A A} / Z_{A}^{2}$ in Fig. 10(a) and that of $Z_{V V+A A} / Z_{P}^{2}$ in Fig. 10(b). Solid lines are tadpole-improved one-loop results in the RI scheme. We observe that the NPR result for $Z_{V V+A A} / Z_{A}^{2}$ has a $p^{2} a^{2}$ dependence opposite to

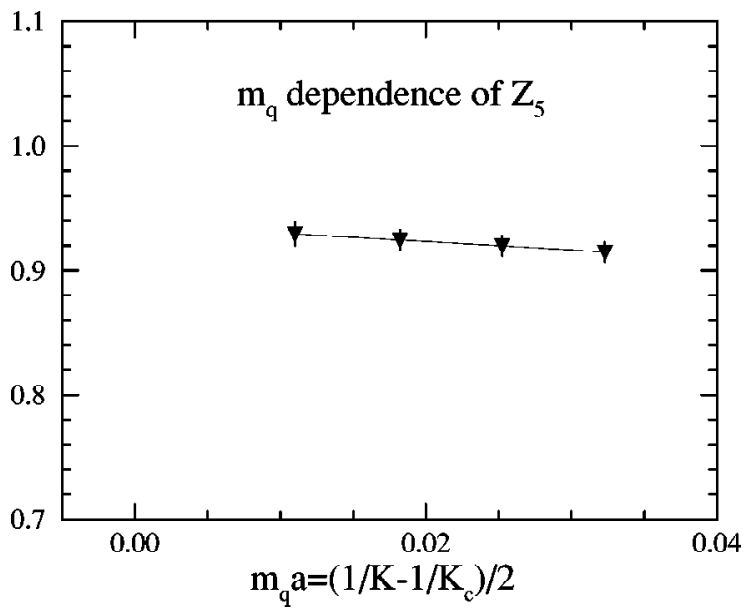

FIG. 7. Quark mass dependence of mixing coefficient $z_{5}$ evaluated at $p^{(*)} \approx 2 \mathrm{GeV}$ using the Ward identity method at $\beta=6.3$. 


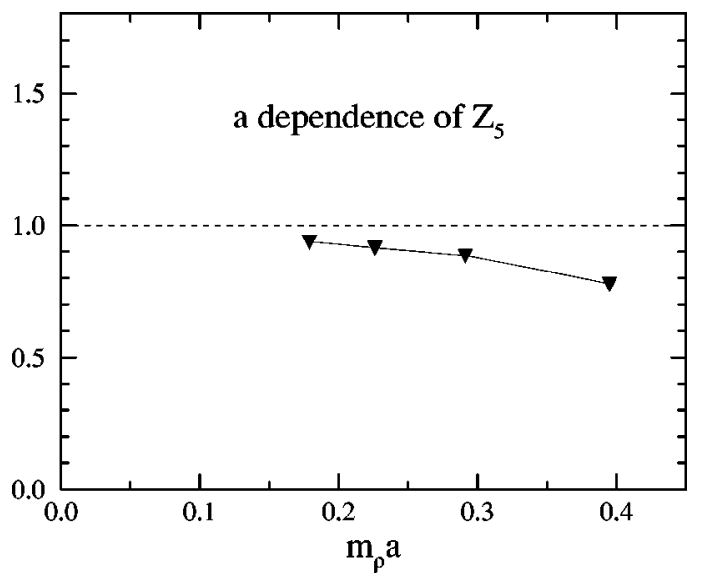

FIG. 8. Lattice spacing dependence of mixing coefficient $z_{5}$ evaluated at $p^{(*)} \approx 2 \mathrm{GeV}$ using the Ward identity method.

that expected from the perturbative estimate. For the ratio $Z_{V V+A A} / Z_{P}^{2}$ the NPR result diverges toward lower momentum.

Application of the NPR method requires the existence of a region $\Lambda_{\mathrm{QCD}} \ll p \ll 1 / a$ where we can keep under control both nonperturbative contaminations and cutoff effects, which is called a "window" in Ref. [6]. We find for $Z_{V V+A A}, Z_{A}, Z_{P}$, and their ratios that the lower bound of the window depends strongly on each operator; we observe $p^{2} a^{2} \sim 0.1$ for $Z_{V V+A A}$ and $p^{2} a^{2} \sim 0.3$ for $Z_{A}$, while it is difficult to find the lower bound for $Z_{P}, Z_{V V+A A} / Z_{A}^{2}$, and

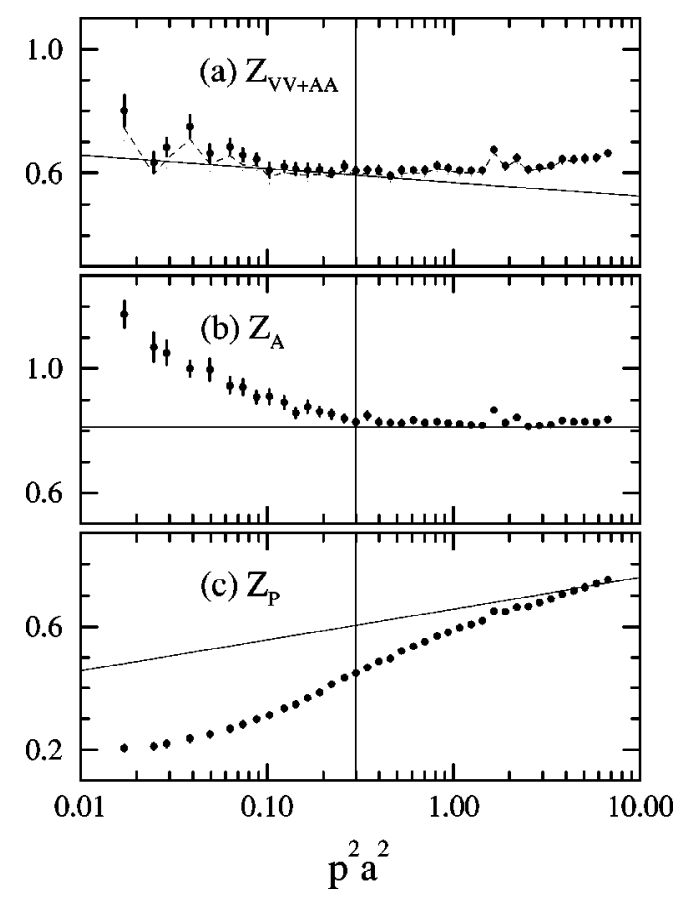

FIG. 9. Renormalization factors (a) $Z_{V V+A A}$, (b) $Z_{A}$, and (c) $Z_{P}$ in the RI scheme obtained with the NPR method as a function of external momentum squared $p^{2} a^{2}$ for $K=0.15034$ at $\beta=6.3$. Transverse lines denote tadpole-improved perturbative estimates. The dotted curve in (a) represents the $\mathcal{O}_{0}$ contribution to $Z_{V V+A A}$. Vertical lines correspond to $p^{(*)} \approx 2 \mathrm{GeV}$.

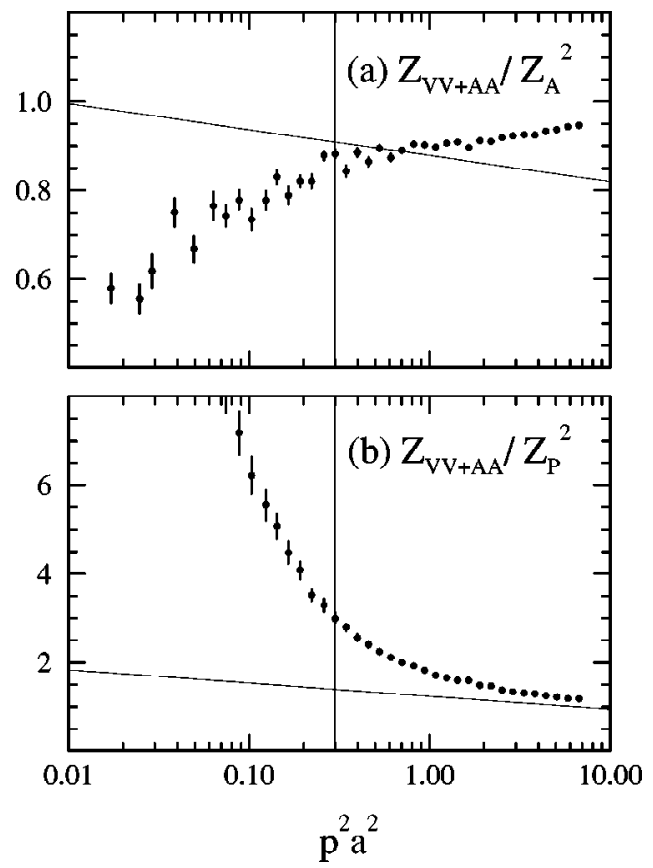

FIG. 10. Ratios of renormalization factors (a) $Z_{V V+A A} / Z_{A}^{2}$ and (b) $Z_{V V+A A} / Z_{P}^{2}$ obtained with the NPR method as a function of external momentum squared $p^{2} a^{2}$ for $K=0.15034$ at $\beta=6.3$. Transverse lines denote tadpole-improved perturbative estimates. Vertical lines correspond to $p^{(*)} \approx 2 \mathrm{GeV}$.

$Z_{V V+A A} / Z_{P}^{2}$. It is not clear to what extent nonperturbative contaminations can be separated out quantitatively. For these reasons we employ the perturbative estimates for $Z_{V V+A A}$, $Z_{A}$, and $Z_{P}$, rather than those of the NPR method, to obtain the $B$ parameter in our final analysis presented in this article. Numerical values of the $B_{K}$ parameter are little affected by this change since the the ratio $Z_{V V+A A} / Z_{A}^{2}$ has a similar value at $p^{(*)}$ among the two methods.

For completeness let us examine the quark mass dependence and the $a$ dependence of $Z_{V V+A A}, Z_{A}$, and $Z_{P}$ taking their results at $p^{(*)}$ (vertical lines in Fig. 9). In Fig. 11 we

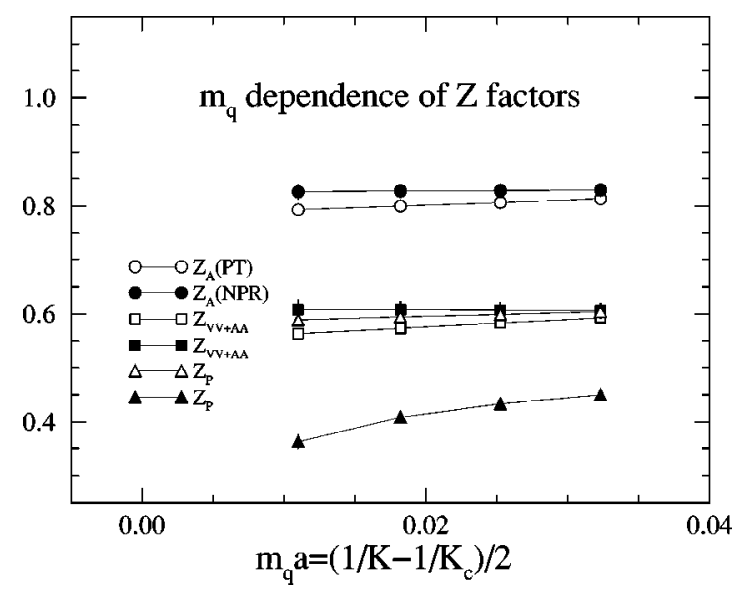

FIG. 11. Quark mass dependence of renormalization factors at $p^{(*)} \approx 2 \mathrm{GeV}$ using the NPR (solid symbols) method at $\beta=6.3$. Open symbols represent tadpole-improved perturbative estimates. 


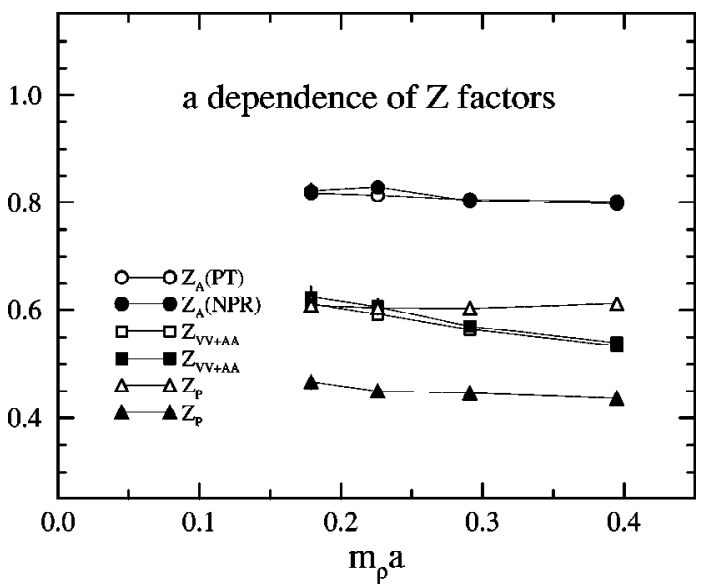

FIG. 12. Comparison of renormalization factors evaluated at $p^{(*)} \approx 2 \mathrm{GeV}$ using the NPR (NPR, solid symbols) method and the tadpole-improved perturbation theory (PT, open symbols) as a function of $m_{\rho} a$.

plot the NPR results for $Z_{V V+A A}, Z_{A}$, and $Z_{P}$ together with tadpole-improved perturbative values as a function of $m_{q}$ $=\left(1 / K-1 / K_{c}\right) / 2$ for the case of $\beta=6.3$. While we observe little quark mass dependence for $Z_{V V+A A}$ and $Z_{A}, Z_{P}$ clearly decreases as the quark mass decreases. Figure 12 shows the $a$ dependence of $Z_{V V+A A}, Z_{A}$, and $Z_{P}$ evaluated at $p^{(*)}$ employing the heaviest quark mass at each $\beta$. The NPR results for $Z_{V V+A A}$ and $Z_{A}$ are consistent with the perturbative ones at four $\beta$ values, while for $Z_{P}$ we observe a large deviation at each $\beta$.

\section{CHIRAL BEHAVIOR}

Let us examine the chiral property of the operator $\hat{\mathcal{O}}_{V V+A A}$ using the matrix element $B_{K}^{P}$ defined in Eq. (64), which vanishes in the chiral limit in the presence of chiral symmetry. In Fig. 13 we show the chiral behavior of $B_{K}^{P}(\mathrm{NDR}, 1 / a)$ for the case of $\beta=6.3$. Numerical values of

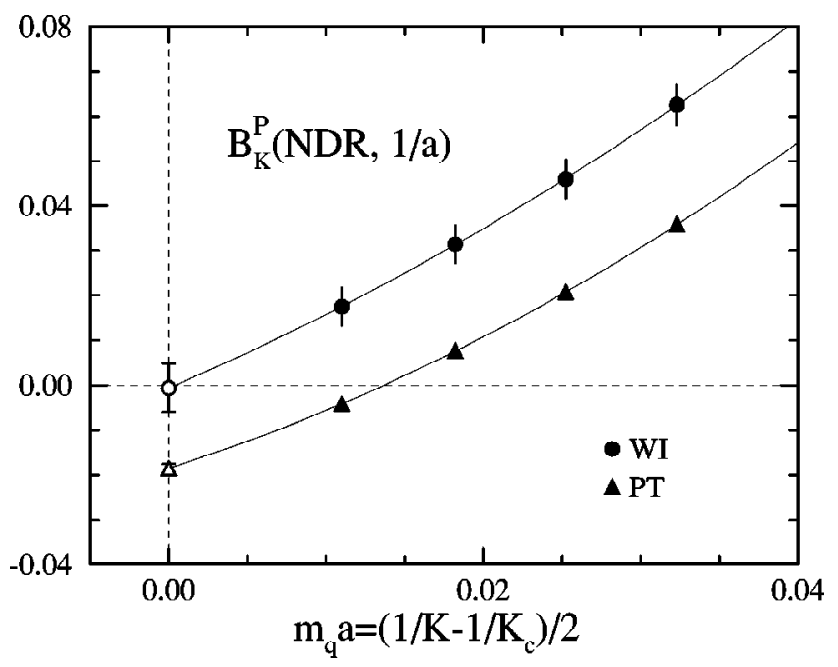

FIG. 13. Test of the chiral behavior of $B_{K}^{P}(\mathrm{NDR}, 1 / a)$ for the Ward identity and perturbative methods at $\beta=6.3$. Solid curves are quadratic extrapolations to the chiral limit.
$B_{K}^{P}(\mathrm{NDR}, 1 / a)$ for the four hopping parameters at each $\beta$ are summarized in Table III. The solid lines represent quadratic extrapolations of the WI and PT results in the bare quark mass $m_{q} a=\left(1 / K-1 / K_{c}\right) / 2$. The extrapolated value at $m_{q}$ $=0$ is consistent with zero, demonstrating a significant improvement of the chiral behavior compared to the perturbative result plotted with triangles.

Figure 14 shows the $p^{2} a^{2}$ dependence of $B_{K}^{P}(\mathrm{NDR}, 1 / a)$ extrapolated to $m_{q}=0$, which are evaluated at each scale $p^{(i)}$. Two solid lines represent the upper and lower bounds of one standard deviation error for the PT result. The values of $B_{K}^{P}(\mathrm{NDR}, 1 / a)$ for the WI method are consistent with zero within error bars in the momentum range $p^{2} a^{2} \lesssim 1$, albeit the errors become larger toward the small momentum region.

We plot in Fig. 15 the values of $B_{K}^{P}(\mathrm{NDR}, 2 \mathrm{GeV})$ in the chiral limit as a function of lattice spacing. The numerical values are given in Table IV. The result for the WI method becomes consistent with zero at the lattice spacing $m_{\rho} a$ $\lesssim 0.3(a \lesssim 0.08 \mathrm{fm})$. In the perturbative approach with the one-loop mixing coefficients, chiral breaking effects are expected to appear primarily as terms of $O(a)$ and secondly as terms of $O\left[a g^{2}(1 / a)\right]$ and $O\left[g^{4}(1 / a)\right]$ for the Wilson quark action. A roughly linear behavior of our results for the perturbative method indicate the leading contribution from an $O(a)$ term. Making a linear extrapolation to the continuum limit $a \rightarrow 0$, we observe that the chiral behavior is recovered. This may suggest that the $O\left[\operatorname{ag}^{2}(1 / a)\right]$ and $O\left[g^{4}(1 / a)\right]$ terms in the mixing coefficients left out in the one-loop treatment are small or accidentally canceled.

\section{RESULTS FOR $B_{K}$}

We now turn to the calculation of $B_{K}(\mathrm{NDR}, 2 \mathrm{GeV})$. In Fig. 16 we present the ratio $R_{A}(t)$ defined in Eq. (84) using the mixing coefficients determined from the Ward identities with the external quark momentum $p^{(*)}$ for the heaviest quark mass $(K=0.15034)$ and the lightest one $(K$ $=0.15131$ ) at $\beta=6.3$. A good plateau is observed in the range $20 \leqq t \leqq 75$. We make a global fit of the ratio $R_{A}(t)$ to a constant over $32 \leqslant t \leqslant 63$ for this data set. The three horizontal lines denote the central value of $B_{K}(\mathrm{NDR}, 1 / a)$ and a one standard deviation error band. We note that the error of the fitted result is roughly equal in magnitude to those of the ratio over the fitted range, while we would usually expect a smaller error for the fitted result. This is because the error of the ratio $R_{A}(t)$ is governed by those of the mixing coefficients $z_{i}(i=1, \ldots, 4)$. Numerical values of $B_{K}(\mathrm{NDR}, 1 / a)$ for the four hopping parameters at each $\beta$ are listed in Table III.

For comparison we also show the results for $R_{A}(t)$ obtained with the perturbative mixing coefficients in Fig. 17. We observe a plateau in the range $30 \leqq t \leqq 65$, which is slightly narrower compared to the WI results. A global fit of $R_{A}(t)$ to a constant choosing the same fitting range as for the WI case yields the value of $B_{K}(\mathrm{NDR}, 1 / a)$ given in Table III. Let us note that the PT results have quite small errors compared to those of the WI method. This is because definite values are taken for the mixing coefficients in the PT method. 
TABLE III. $B$ parameters obtained with the WI, $\mathrm{WI}_{\mathrm{VS}}$, and PT methods for $\beta=5.9-6.5$ in quenched QCD. Operators are renormalized at $1 / a$ in the NDR scheme.

\begin{tabular}{|c|c|c|c|c|c|c|}
\hline \multirow[b]{2}{*}{$\beta$} & \multirow[b]{2}{*}{$K$} & \multicolumn{3}{|c|}{$B_{K}(\mathrm{NDR}, 1 / a)$} & \multicolumn{2}{|c|}{$B_{K}^{P}(\mathrm{NDR}, 1 / a)$} \\
\hline & & WI & $\mathrm{WI}_{\mathrm{VS}}$ & PT & WI & PT \\
\hline \multirow[t]{4}{*}{5.9} & 0.15862 & $0.270(75)$ & $0.108(26)$ & $-0.631(13)$ & $0.0076(21)$ & $-0.01767(30)$ \\
\hline & 0.15785 & $0.507(39)$ & $0.259(13)$ & $-0.0728(56)$ & $0.0236(18)$ & $-0.00340(25)$ \\
\hline & 0.15708 & $0.620(26)$ & $0.3617(82)$ & $0.1937(38)$ & $0.0408(17)$ & $0.01274(26)$ \\
\hline & 0.15632 & $0.687(20)$ & $0.4348(60)$ & $0.3501(32)$ & $0.0585(17)$ & $0.02977(30)$ \\
\hline \multirow[t]{4}{*}{6.1} & 0.15428 & $0.62(14)$ & $0.226(33)$ & $-0.416(24)$ & $0.0156(34)$ & $-0.01039(49)$ \\
\hline & 0.15381 & $0.686(75)$ & $0.331(20)$ & $-0.044(13)$ & $0.0293(32)$ & $0.00186(57)$ \\
\hline & 0.15333 & $0.720(51)$ & $0.406(14)$ & $0.257(10)$ & $0.0439(31)$ & $0.01565(66)$ \\
\hline & 0.15287 & $0.747(39)$ & $0.461(11)$ & $0.3813(86)$ & $0.0585(31)$ & $0.02985(74)$ \\
\hline \multirow[t]{4}{*}{6.3} & 0.15131 & $0.66(16)$ & $0.286(41)$ & $-0.157(22)$ & $0.0175(42)$ & $-0.00418(53)$ \\
\hline & 0.15098 & $0.713(92)$ & $0.387(26)$ & $0.173(12)$ & $0.0315(41)$ & $0.00765(56)$ \\
\hline & 0.15066 & $0.745(68)$ & $0.455(19)$ & $0.3362(96)$ & $0.0460(43)$ & $0.02072(63)$ \\
\hline & 0.15034 & $0.775(54)$ & $0.511(16)$ & $0.4449(87)$ & $0.0625(45)$ & $0.03586(75)$ \\
\hline \multirow[t]{4}{*}{6.5} & 0.14925 & $0.69(39)$ & $0.212(87)$ & $-0.338(61)$ & $0.0115(65)$ & $-0.00564(87)$ \\
\hline & 0.14901 & $0.65(17)$ & $0.327(46)$ & $0.148(25)$ & $0.0223(59)$ & $0.00512(92)$ \\
\hline & 0.14877 & $0.67(11)$ & $0.406(33)$ & $0.329(20)$ & $0.0348(58)$ & $0.0171(12)$ \\
\hline & 0.14853 & $0.699(82)$ & $0.467(24)$ & $0.440(15)$ & $0.0483(57)$ & $0.0304(12)$ \\
\hline
\end{tabular}

In Fig. 18 a representative result for the contribution of each operator $\mathcal{O}_{i}(i=0, \ldots, 4)$ to $B_{K}(\mathrm{NDR}, 1 / a)$ is shown as a function of the external quark momentum for the case of $K=0.15034$ at $\beta=6.3$, which is obtained by fitting the ratio $R_{A}^{i}(t) \quad(i=0, \ldots, 4)$ of Eq. (89) with a constant over the same fitting range as for $R_{A}(t)$. The contributions of mixed operators are nearly independent of the external quark momentum in the range $p^{2} a^{2} \lesssim 1.0$. An important observation is that the value of $B_{K}(\mathrm{NDR}, 1 / a)$ results from large cancellations between the amplitudes of the mixing operators $z_{i} \mathcal{O}_{i}$ $(i=1, \ldots, 4)$, each having a magnitude comparable to or

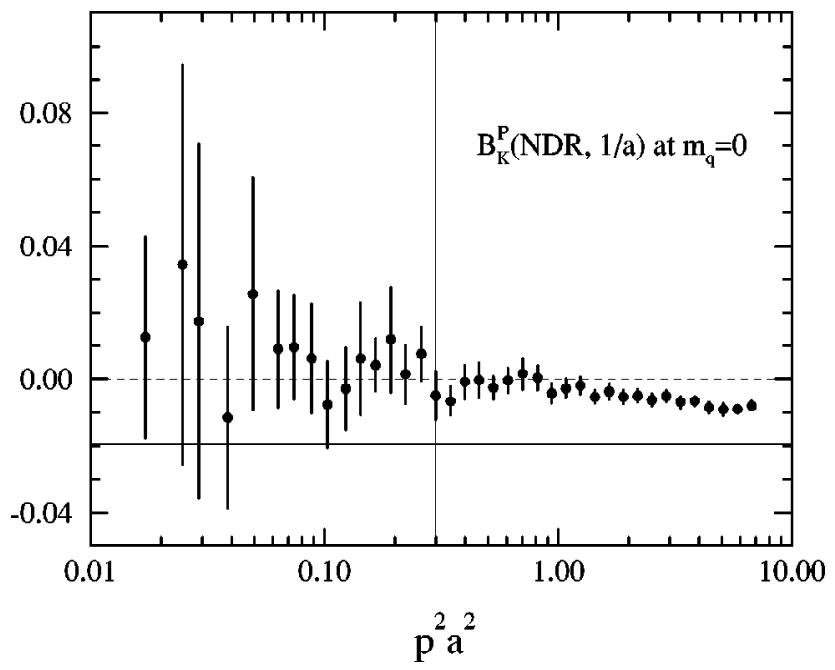

FIG. 14. Dependence of $B_{K}^{P}(\mathrm{NDR}, 1 / a)$ in the chiral limit on the external momentum squared $p^{2} a^{2}$ at $\beta=6.3$. Horizontal solid lines represent the upper and lower bound of one standard deviation error for tadpole-improved perturbative result in the chiral limit. The vertical line corresponds to $p^{(*)} \approx 2 \mathrm{GeV}$. larger than that of $\mathcal{O}_{0}$. This is the essential reason why calculations of $B_{K}$ with the Wilson quark action is difficult; the mixing coefficients have to be known accurately including higher order effects both in the coupling constant and the lattice spacing.

We show the quark mass dependence of $B_{K}(\mathrm{NDR}, 1 / a)$ for $\beta=6.3$ in Fig. 19. We observe that the results for the PT method seem to diverge toward the chiral limit, while those for the WI method stay finite. We expect a different quark mass dependence for the WI and PT results:

$$
B_{K}(\mathrm{NDR}, 1 / a)=A^{\mathrm{WI}}+B^{\mathrm{WI}} m_{q}+C^{\mathrm{WI}} m_{q}^{2} \text { for } \mathrm{WI},
$$

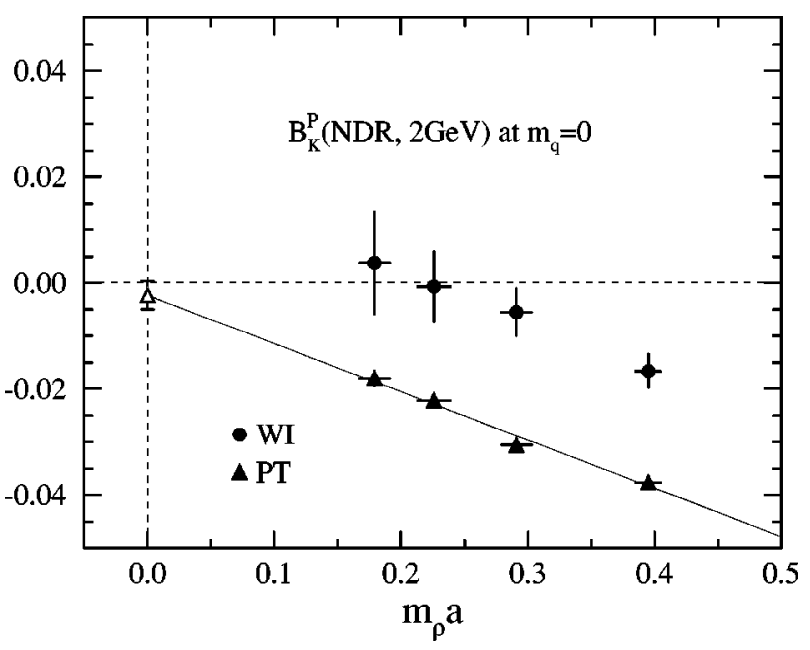

FIG. 15. $B_{K}^{P}(\mathrm{NDR}, 2 \mathrm{GeV})$ at $m_{q}=0$ for the Ward identity and perturbative methods as a function of $a$. The solid line is a linear extrapolation of the perturbative results to the continuum limit. 
TABLE IV. Results for $B_{K}(\mathrm{NDR}, 2 \mathrm{GeV})$ obtained with the WI, $\mathrm{WI}_{\mathrm{VS}}$, and PT methods as a function of $\beta$. Values of $B_{K}^{P}(\mathrm{NDR}, 2 \mathrm{GeV})$ in the chiral limit for the WI and PT methods are also given.

\begin{tabular}{|c|c|c|c|c|c|c|}
\hline$\beta$ & & 5.9 & 6.1 & 6.3 & 6.5 & $a=0$ \\
\hline \multirow[t]{3}{*}{$B_{K}(\mathrm{NDR}, 2 \mathrm{GeV})$} & WI & $+0.360(60)$ & $+0.66(11)$ & $+0.71(12)$ & $+0.69(19)$ & \\
\hline & $\mathrm{WI}_{\mathrm{VS}}$ & $+0.162(20)$ & $+0.278(27)$ & $+0.346(35)$ & $+0.347(55)$ & $+0.562(66)$ \\
\hline & PT & $-0.391(13)$ & $-0.167(20)$ & $+0.037(19)$ & $+0.180(36)$ & $+0.622(69)$ \\
\hline \multirow{2}{*}{$\left.B_{K}^{P}(\mathrm{NDR}, 2 \mathrm{GeV})\right|_{m_{q}=0}$} & WI & $-0.0166(32)$ & $-0.0055(45)$ & $-0.0007(66)$ & $+0.0038(96)$ & \\
\hline & PT & $-0.03761(67)$ & $-0.03055(90)$ & $-0.0222(11)$ & $-0.0180(15)$ & $-0.0023(27)$ \\
\hline
\end{tabular}

$$
B_{K}(\mathrm{NDR}, 1 / a)=\frac{A^{\mathrm{PT}}}{m_{q}}+B^{\mathrm{PT}}+C^{\mathrm{PT}} m_{q} \text { for } \mathrm{PT},
$$

where $A, B$, and $C$ are unknown constants. These functional forms are based on the following assumption for the chiral behavior of the matrix elements near the chiral limit:

$$
\begin{gathered}
\left\langle\bar{K}^{0}\left|\hat{\mathcal{O}}_{V V+A A}\right| K^{0}\right\rangle \propto m_{q} \text { for } \mathrm{WI}, \\
\left\langle\bar{K}^{0}\left|\hat{\mathcal{O}}_{V V+A A}\right| K^{0}\right\rangle \propto \text { const } \text { for } \mathrm{PT}, \\
\left\langle 0\left|\hat{A}_{\mu}\right| K^{0}\right\rangle \propto \sqrt{m_{q}} .
\end{gathered}
$$

For the WI and PT methods we interpolate the data at the four hopping parameters with the forms (96) and (97), respectively, to $m_{s} / 2$ and obtain the value of $B_{K}(\mathrm{NDR}, 1 / a)$ at the physical point.

We summarize our final results for $B_{K}(\mathrm{NDR}, 2 \mathrm{GeV})$ in Table IV, whose $a$ dependence is illustrated in Fig. 20. The method based on the Ward identity gives a value well con-

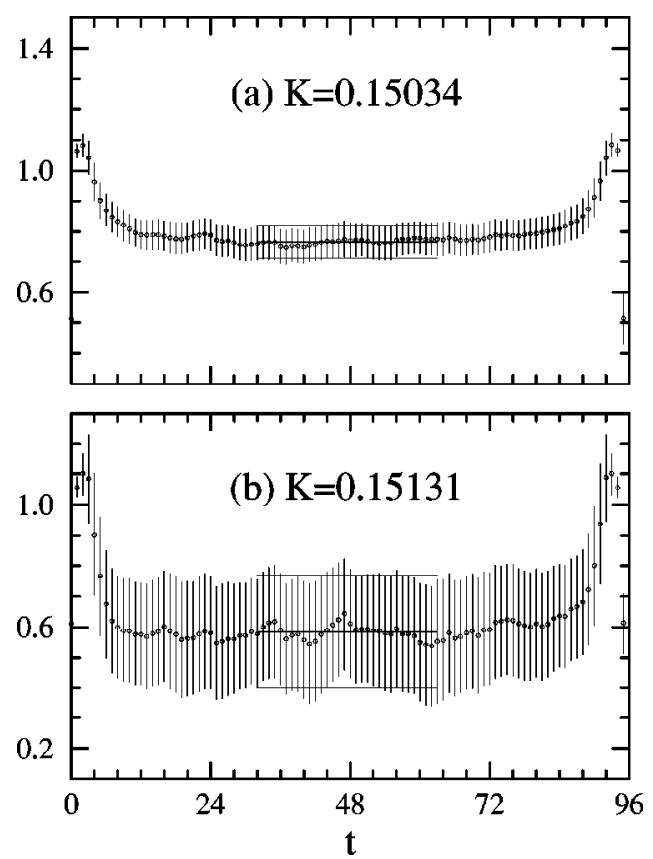

FIG. 16. Ratio $R_{A}(t)$ using mixing coefficients determined by the Ward identity method for (a) $K=0.15034$ and (b) $K=0.15131$ at $\beta=6.3$. Solid lines denote the fitted result and a one standard deviation error band. vergent from a lattice spacing of $m_{\rho} a \approx 0.3$. Unfortunately the large errors do not allow us to take a linear extrapolation to the continuum limit. We may instead take a constant fit of the three results at smaller lattice spacings $\left(a^{-1}\right.$ $=2.7-4.3 \mathrm{GeV})$ and find $B_{K}(\mathrm{NDR}, 2 \mathrm{GeV})=0.68(7)$, which is our best estimate for the WI method.

Since the origin of the large error is traced to that of the mixing coefficients, we attempt to develop an alternative method, in which the denominator of Eq. (59) is estimated with the vacuum saturation of the operator $\hat{\mathcal{O}}_{V V+A A}$ constructed by the WI method:

$$
B_{K}^{\mathrm{VS}}(\mathrm{NDR}, 1 / a)=\frac{\left\langle\bar{K}^{0}\left|\hat{\mathcal{O}}_{V V+A A}\right| K^{0}\right\rangle}{Z_{A}^{2}\left\langle\bar{K}^{0}\left|\mathcal{O}_{V V+A A}\right| K^{0}\right\rangle_{\mathrm{VS}}},
$$

where in terms of Eq. (42) the vacuum saturation of $\hat{\mathcal{O}}_{V V+A A}$ is rewritten as

$$
\left\langle\bar{K}^{0}\left|\mathcal{O}_{V V+A A}\right| K^{0}\right\rangle_{\mathrm{VS}}=\sum_{i=0}^{4} z_{i}\left\langle\bar{K}^{0}\left|\mathcal{O}_{i}\right| K^{0}\right\rangle_{\mathrm{VS}},
$$

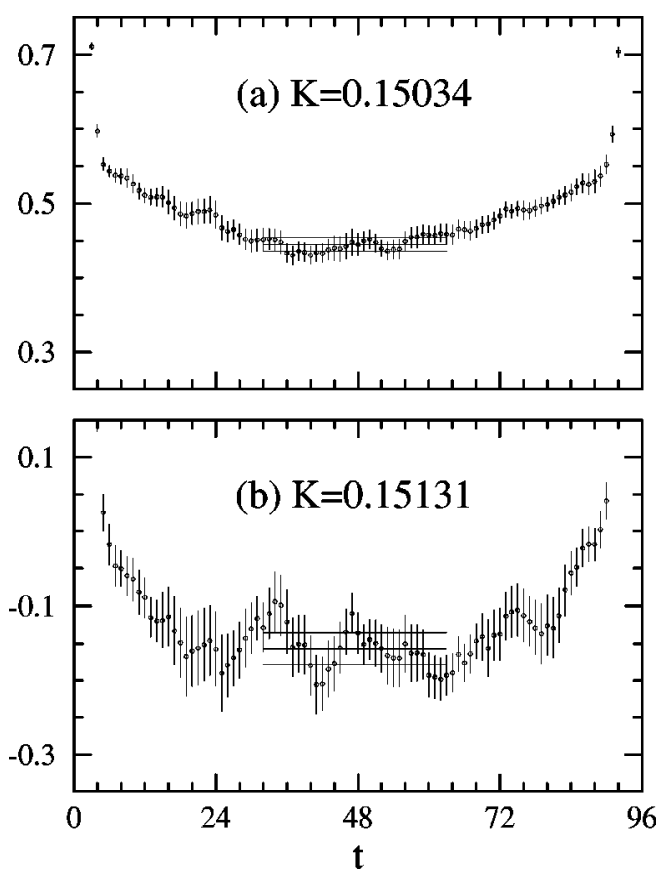

FIG. 17. Same as Fig. 16 for the perturbative method. 


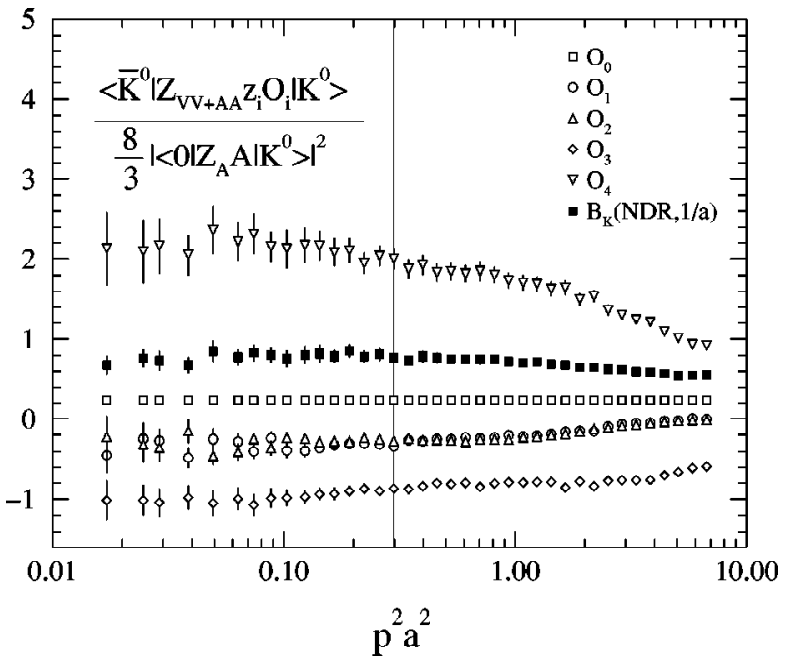

FIG. 18. Contributions of the operators $\mathcal{O}_{i}(i=0, \ldots, 4)$ to $B_{K}(\mathrm{NDR}, 1 / a)$ with $z_{i}$ determined by the Ward identity method for $K=0.15034$ at $\beta=6.3$. Vertical line corresponds to $p^{(*)} \approx 2 \mathrm{GeV}$.

with

$$
\begin{aligned}
\left\langle\bar{K}^{0}\left|\mathcal{O}_{0}\right| K^{0}\right\rangle_{\mathrm{VS}}= & \frac{8}{3}\left\langle\bar{K}^{0}|A| 0\right\rangle\left\langle 0|A| K^{0}\right\rangle, \\
\left\langle\bar{K}^{0}\left|\mathcal{O}_{1}\right| K^{0}\right\rangle_{\mathrm{VS}}= & \frac{8}{3}\left\langle\bar{K}^{0}|P| 0\right\rangle\left\langle 0|P| K^{0}\right\rangle, \\
\left\langle\bar{K}^{0}\left|\mathcal{O}_{2}\right| K^{0}\right\rangle_{\mathrm{VS}}= & \frac{4}{3}\left\langle\bar{K}^{0}|P| 0\right\rangle\left\langle 0|P| K^{0}\right\rangle, \\
\left\langle\bar{K}^{0}\left|\mathcal{O}_{3}\right| K^{0}\right\rangle_{\mathrm{VS}}= & -\frac{4}{3}\left\langle\bar{K}^{0}|A| 0\right\rangle\left\langle 0|A| K^{0}\right\rangle \\
& -\frac{8}{3}\left\langle\bar{K}^{0}|P| 0\right\rangle\left\langle 0|P| K^{0}\right\rangle,
\end{aligned}
$$

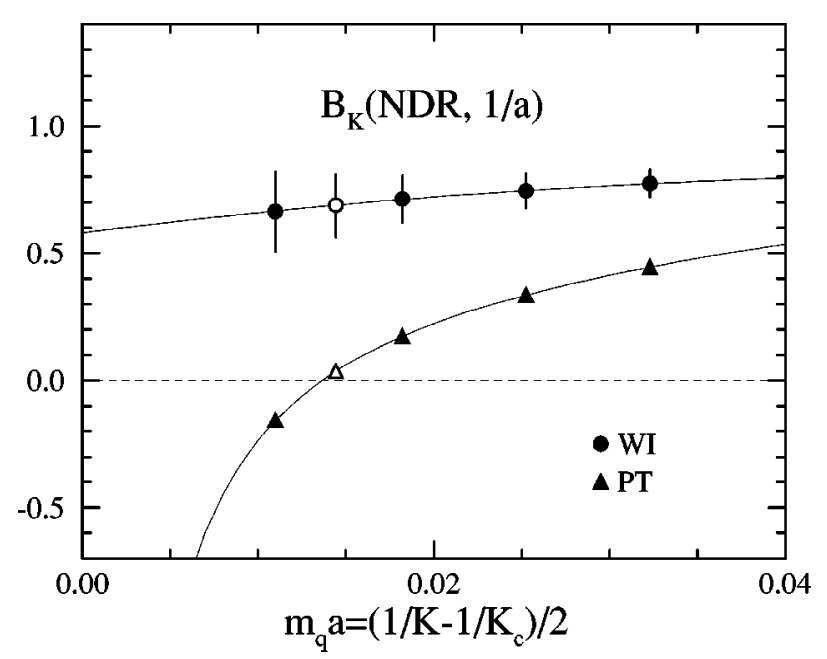

FIG. 19. Quark mass dependence of $B_{K}(\mathrm{NDR}, 1 / a)$ for the Ward identity method and the tadpole-improved perturbative one at $\beta=6.3$. Open symbols are interpolations of data to $m_{s} / 2$.

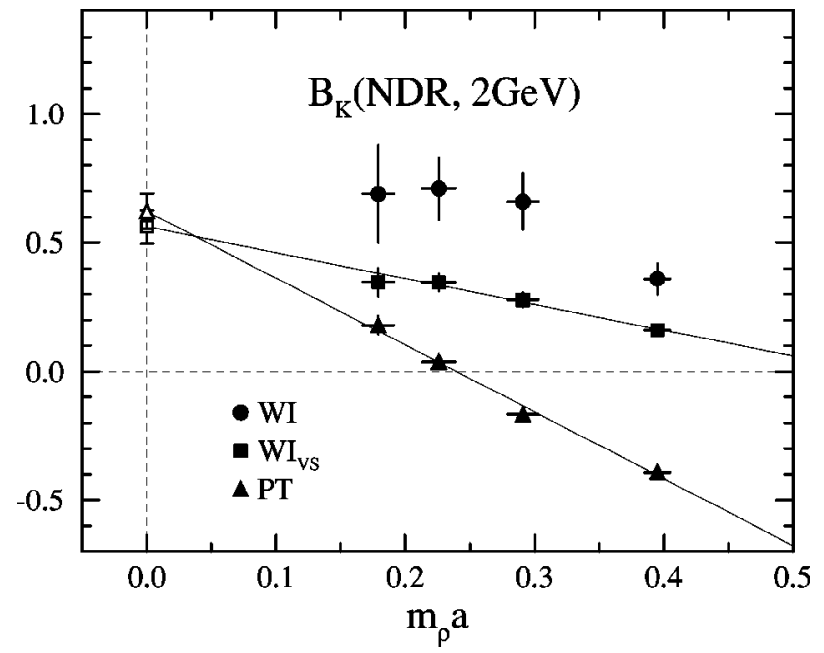

FIG. 20. $B_{K}(\mathrm{NDR}, 2 \mathrm{GeV})$ plotted as a function of $m_{\rho} a$ for the $\mathrm{WI}, \mathrm{WI}_{\mathrm{VS}}$, and PT methods. Solid lines show linear extrapolations to the continuum limit.

$$
\begin{aligned}
\left\langle\bar{K}^{0}\left|\mathcal{O}_{4}\right| K^{0}\right\rangle_{\text {VS }}= & -\frac{8}{3}\left\langle\bar{K}^{0}|A| 0\right\rangle\left\langle 0|A| K^{0}\right\rangle+\frac{16}{3}\left\langle\bar{K}^{0}|P| 0\right\rangle \\
& \times\left\langle 0|P| K^{0}\right\rangle .
\end{aligned}
$$

We refer to this as the $\mathrm{WI}_{\mathrm{VS}}$ method, with which the fluctuations in the numerator are expected to largely cancel against those in the denominator. In fact, errors are substantially reduced with the $\mathrm{WI}_{\mathrm{VS}}$ method as apparent in Fig. 20. The cost is that the correct chiral behavior of the denominator is not respected at a finite lattice spacing due to the contributions of the pseudoscalar matrix element. This contribution brings the $\mathrm{WI}_{\mathrm{VS}}$ result to disagree with WI at a finite lattice spacing, but the discrepancy should vanish in the continuum limit. A linear extrapolation of the $\mathrm{WI}_{\mathrm{VS}}$ results in $a$ yields $B_{K}(\mathrm{NDR}, 2 \mathrm{GeV})=0.562(66)$.

This linear extrapolation, however, involves a systematic uncertainty arising from the chiral symmetry breaking term $c_{P}\left|\left\langle 0|P| K^{0}\right\rangle\right|^{2}$ in the denominator, where $c_{P}=8 / 3 z_{1}+4 / 3 z_{2}$

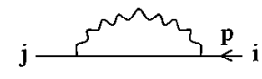

(a)

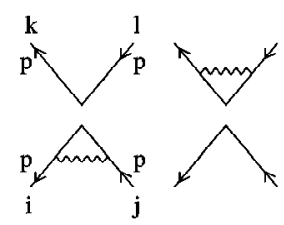

(c)

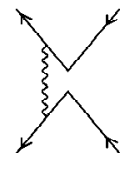

(e)

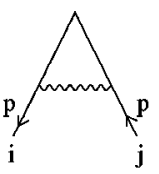

(b)

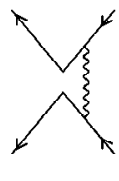

(f)

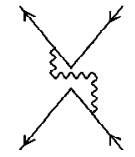

(g)

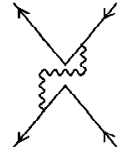

(h)
FIG. 21. One-loop diagrams for (a) quark self-energy, (b) vertex correction for the quark bilinear operator, and (c)-(h) vertex corrections for the four-quark operator. $p$ denotes an off-shell momentum for the external quark state, and $i, j, k$, and $l$ label color indices. 
TABLE V. Results for $\Sigma^{(1)}(p)$ and $\Lambda_{\Gamma}^{(1)}(p)$. In the entries labeled "color" the color factors are given with the use of $C_{F}=4 / 3$ and $N_{c}=3$. In the third column $\mathrm{F}$ refers to the results in the Feynman gauge $\lambda$ $=1$. L refers to the coefficients of the gauge parameter $-(1-\lambda)$ for the results in a general gauge. Divergent part is expressed as $1 / \widetilde{\boldsymbol{\epsilon}} \equiv 1 / \boldsymbol{\epsilon}-\gamma_{\mathrm{E}}+\ln 4 \pi \cdot \boldsymbol{p}^{L} \otimes \boldsymbol{p}^{L}$ represents $p_{\mu} \gamma_{\mu}\left(1-\gamma_{5}\right) \otimes p_{\nu} \gamma_{\nu}\left(1-\gamma_{5}\right)$. The definition of evanescent operators $E$ are given in Eqs. (A6)-(A8).

(a) NDR

\begin{tabular}{|c|c|c|c|}
\hline$\Sigma^{(1)}(p) /(i p)$ & $\begin{array}{l}\text { color } \\
\text { (a) } \\
\text { (a) }\end{array}$ & $\begin{array}{l}\mathrm{F} \\
\mathrm{L}\end{array}$ & $\begin{array}{l}C_{F} \delta_{i j} \\
-1 / \widetilde{\boldsymbol{\epsilon}}-\ln \left|\mu^{2} / p^{2}\right|-1 \\
-1 / \widetilde{\boldsymbol{\epsilon}}-\ln \left|\mu^{2} / p^{2}\right|-1\end{array}$ \\
\hline$\Lambda_{\gamma_{\mu} \gamma_{5}}^{(1)}(p)$ & $\begin{array}{l}\text { color } \\
\text { (b) } \\
\text { (b) }\end{array}$ & $\mathrm{L}$ & $\begin{array}{c}C_{F} \delta_{i j} \\
\gamma_{\mu} \gamma_{5}\left(1 / \widetilde{\boldsymbol{\epsilon}}+\ln \left|\mu^{2} / p^{2}\right|+1\right)-2 p_{\mu} \phi \gamma_{5} / p^{2} \\
\gamma_{\mu} \gamma_{5}\left(1 / \widetilde{\boldsymbol{\epsilon}}+\ln \left|\mu^{2} / p^{2}\right|+1\right)-2 p_{\mu} \phi \gamma_{5} / p^{2}\end{array}$ \\
\hline$\Lambda_{\gamma_{5}}^{(1)}(p)$ & $\begin{array}{l}\text { color } \\
\text { (b) } \\
\text { (b) }\end{array}$ & $\begin{array}{l}\mathrm{F} \\
\mathrm{L}\end{array}$ & $\begin{array}{c}C_{F} \delta_{i j} \\
\gamma_{5}\left(4 / \widetilde{\boldsymbol{\epsilon}}+4 \ln \left|\mu^{2} / p^{2}\right|+6\right) \\
\gamma_{5}\left(1 / \widetilde{\boldsymbol{\epsilon}}+\ln \left|\mu^{2} / p^{2}\right|+2\right)\end{array}$ \\
\hline$\Lambda_{\gamma_{\mu}^{L} \otimes \gamma_{\mu}^{L}}^{(1)}(p)$ & $\begin{array}{l}\text { color } \\
\text { (c), (d) } \\
\text { (c), (d) } \\
\text { color } \\
\text { (e), (f) } \\
\text { (e), (f) } \\
\text { color } \\
\text { (g), (h) } \\
\text { (g), (h) }\end{array}$ & $\begin{array}{l}\mathrm{F} \\
\mathrm{L}\end{array}$ & $\begin{array}{c}C_{F} \delta_{i j} \delta_{k l} / 2+\left(\delta_{i j} \delta_{k l}-\delta_{i l} \delta_{k j} / N_{c}\right) / 4 \\
\gamma_{\mu}^{L} \otimes \gamma_{\mu}^{L}\left(1 / \widetilde{\boldsymbol{\epsilon}}+\ln \left|\mu^{2} / p^{2}\right|+1\right)-2 p^{L} \otimes p^{L} / p^{2} \\
\gamma_{\mu}^{L} \otimes \gamma_{\mu}^{L}\left(1 / \widetilde{\boldsymbol{\epsilon}}+\ln \left|\mu^{2} / p^{2}\right|+1\right)-2 p^{L} \otimes p^{L} / p^{2} \\
\left(\delta_{i l} \delta_{k j}-\delta_{i j} \delta_{k l} / N_{c}\right) / 4+\left(\delta_{i j} \delta_{k l}-\delta_{i l} \delta_{k j} / N_{c}\right) / 4 \\
\gamma_{\mu}^{L} \otimes \gamma_{\mu}^{L}\left(-4 / \widetilde{\boldsymbol{\epsilon}}-4 \ln \left|\mu^{2} / p^{2}\right|-9+16 \ln 2\right)+E_{\gamma_{\mu} \otimes \gamma_{\mu}}^{\mathrm{NDR}} / \epsilon \\
\gamma_{\mu}^{L} \otimes \gamma_{\mu}^{L}\left(-1 / \widetilde{\boldsymbol{\epsilon}}-\ln \left|\mu^{2} / p^{2}\right|-2+4 \ln 2\right) \\
\left(\delta_{i l} \delta_{k j}-\delta_{i j} \delta_{k l} / N_{c}\right) / 4+C_{F} \delta_{i l} \delta_{k j} / 2 \\
\gamma_{\mu}^{L} \otimes \gamma_{\mu}^{L}\left(1 / \widetilde{\boldsymbol{\epsilon}}+\ln \left|\mu^{2} / p^{2}\right|\right)+2 p^{L} \otimes p^{L} / p^{2}+E_{\gamma_{\mu}^{L} \otimes \gamma_{\mu}}^{\mathrm{NDR}} / \epsilon \\
\gamma_{\mu}^{L} \otimes \gamma_{\mu}^{L}\left(1 / \widetilde{\boldsymbol{\epsilon}}+\ln \left|\mu^{2} / p^{2}\right|\right)+2 p^{L} \otimes p^{L} / p^{2}\end{array}$ \\
\hline
\end{tabular}

(b) DRED

\begin{tabular}{|c|c|c|c|}
\hline$\Sigma^{(1)}(p) /(i p p)$ & $\begin{array}{l}\text { color } \\
\text { (a) } \\
\text { (a) }\end{array}$ & $\begin{array}{l}\mathrm{F} \\
\mathrm{L}\end{array}$ & $\begin{array}{l}\quad C_{F} \delta_{i j} \\
-1 / \widetilde{\boldsymbol{\epsilon}}-\ln \left|\mu^{2} / p^{2}\right|-2 \\
-1 / \widetilde{\boldsymbol{\epsilon}}-\ln \left|\mu^{2} / p^{2}\right|-1\end{array}$ \\
\hline$\Lambda_{\gamma_{\mu} \gamma_{5}}^{(1)}(p)$ & $\begin{array}{l}\text { color } \\
\text { (b) } \\
\text { (b) }\end{array}$ & $\begin{array}{l}\mathrm{F} \\
\mathrm{L}\end{array}$ & $\begin{array}{c}C_{F} \delta_{i j} \\
\gamma_{\mu} \gamma_{5}\left(1 / \widetilde{\boldsymbol{\epsilon}}+\ln \left|\mu^{2} / p^{2}\right|+5 / 2\right)-2 p_{\mu} \not p \gamma_{5} / p^{2}-E_{\gamma_{\mu} \gamma_{5}}^{\mathrm{DRED}} / \epsilon \\
\gamma_{\mu} \gamma_{5}\left(1 / \tilde{\boldsymbol{\epsilon}}+\ln \left|\mu^{2} / p^{2}\right|+1\right)-2 p_{\mu} \not \gamma_{5} / p^{2}\end{array}$ \\
\hline$\Lambda_{\gamma_{5}}^{(1)}(p)$ & $\begin{array}{l}\text { color } \\
\text { (b) } \\
\text { (b) }\end{array}$ & $\begin{array}{l}\mathrm{F} \\
\mathrm{L}\end{array}$ & $\begin{array}{c}C_{F} \delta_{i j} \\
\gamma_{5}\left(4 / \widetilde{\boldsymbol{\epsilon}}+4 \ln \left|\mu^{2} / p^{2}\right|+8\right) \\
\gamma_{5}\left(1 / \widetilde{\boldsymbol{\epsilon}}+\ln \left|\mu^{2} / p^{2}\right|+2\right)\end{array}$ \\
\hline$\Lambda_{\gamma_{\mu}^{L} \otimes \gamma_{\mu}^{L}}^{(1)}(p)$ & $\begin{array}{l}\text { color } \\
\text { (c), (d) } \\
\text { (c), (d) } \\
\text { color } \\
\text { (e), (f) } \\
\text { (e), (f) } \\
\text { color } \\
\text { (g), (h) } \\
\text { (g), (h) }\end{array}$ & $\begin{array}{l}\mathrm{F} \\
\mathrm{L}\end{array}$ & $\begin{array}{c}C_{F} \delta_{i j} \delta_{k l} / 2+\left(\delta_{i j} \delta_{k l}-\delta_{i l} \delta_{k j} / N_{c}\right) / 4 \\
\gamma_{\mu}^{L} \otimes \gamma_{\mu}^{L}\left(1 / \widetilde{\boldsymbol{\epsilon}}+\ln \left|\mu^{2} / p^{2}\right|+5 / 2\right)-2 p^{L} \otimes p^{L} / p^{2}-E_{\gamma_{\mu}^{L} \otimes \gamma_{\mu}^{L} / \epsilon}^{\mathrm{DRED}} \\
\gamma_{\mu}^{L} \otimes \gamma_{\mu}^{L}\left(1 / \widetilde{\boldsymbol{\epsilon}}+\ln \left|\mu^{2} / p^{2}\right|+1\right)-2 p^{L} \otimes p^{L} / p^{2} \\
\left(\delta_{i l} \delta_{k j}-\delta_{i j} \delta_{k l} / N_{c}\right) / 4+\left(\delta_{i j} \delta_{k l}-\delta_{i l} \delta_{k j} / N_{c}\right) / 4 \\
\gamma_{\mu}^{L} \otimes \gamma_{\mu}^{L}\left(-4 / \widetilde{\boldsymbol{\epsilon}}-4 \ln \left|\mu^{2} / p^{2}\right|-8+16 \ln 2\right) \\
\gamma_{\mu}^{L} \otimes \gamma_{\mu}^{L}\left(-1 / \widetilde{\boldsymbol{\epsilon}}-\ln \left|\mu^{2} / p^{2}\right|-2+4 \ln 2\right) \\
\left(\delta_{i l} \delta_{k j}-\delta_{i j} \delta_{k l} / N_{c}\right) / 4+C_{F} \delta_{i l} \delta_{k j} / 2 \\
\gamma_{\mu}^{L} \otimes \gamma_{\mu}^{L}\left(1 / \widetilde{\boldsymbol{\epsilon}}+\ln \left|\mu^{2} / p^{2}\right|+3 / 2\right)+2 p^{L} \otimes p^{L} / p^{2}+E_{\gamma_{\mu}^{L} \otimes \gamma_{\mu}^{L} / \epsilon}^{\mathrm{DRED}} \\
\gamma_{\mu}^{L} \otimes \gamma_{\mu}^{L}\left(1 / \widetilde{\boldsymbol{\epsilon}}+\ln \left|\mu^{2} / p^{2}\right|\right)+2 p^{L} \otimes p^{L} / p^{2}\end{array}$ \\
\hline
\end{tabular}

$-8 / 3 z_{3}+16 / 3 z_{4}$ from Eqs. (104)-(107). Perturbative contributions to $c_{P}$ starts at two-loop order of $O\left[g^{4}(1 / a)\right]$ as can be checked from the one-loop expressions for $z_{i}$ ( $i$ $=1, \ldots, 4)$ in Eq. (53). Since the matrix element $\left\langle 0|P| K^{0}\right\rangle$ diverges in proportion to $\left[g^{2}(1 / a)\right]^{-4 / 11}$ due to the anomalous dimension of the pseudoscalar operator $P$, $c_{P}\left|\left\langle 0|P| K^{0}\right\rangle\right|^{2}$ receives contributions of form $\left[g^{2}(1 / a)\right]^{14 / 11}$ which diminishes only as a fractional power of $1 / \log a$. To 
assess the systematic error associated with this effect, we estimate the two-loop contribution to $c_{P}$ by squaring the typical magnitude of the one-loop terms in $z_{i}$ : e.g., $\left|z_{i}^{\text {one-loop }}\left[\alpha_{\overline{\mathrm{MS}}}(1 / a)\right]\right| \lesssim 0.08$ at $\beta=5.9$ from Fig. 5 . We also estimate

$$
\frac{\left\langle 0|P| K^{0}\right\rangle}{\left\langle 0|A| K^{0}\right\rangle}=\frac{m_{K}}{m_{d}+m_{s}} \approx 5
$$

from the PCAC (partial conservation of axial vector current) relation [23], which yields $c_{P}\left|\left\langle 0|P| K^{0}\right\rangle\right|^{2} /(8 / 3) /$ $\left|\left\langle 0|A| K^{0}\right\rangle\right|^{2} \lesssim 0.4$. Since $\alpha_{\overline{\mathrm{MS}}}(1 / a)^{14 / 11}$ decreases by $30 \%$ between $\beta=5.9-6.5$, over which $a$ decreases by a factor 2 , this fraction should reduce to $\approx 0.16$ after taking the continuum limit. Taking account of uncertainties in the choice of coupling constant and the mixing coefficients at the two-loop level, we estimate the chiral symmetry breaking contribution of the pseudoscalar density that survives after a continuum extrapolation linear in $a$ to be $\$ 20 \%$. We conclude $B_{K}(\mathrm{NDR}, 2 \mathrm{GeV})=0.56(7)(11)$ for the $\mathrm{WI}_{\mathrm{Vs}}$ method.

An interesting point in Fig. 20 is that the perturbative calculation (PT), which gives a completely "wrong value" at $a \neq 0$, yields the correct result for $B_{K}$, when extrapolated to the continuum limit $a=0$. This is a long extrapolation from negative to positive, but the linearly extrapolated value $B_{K}(\mathrm{NDR}, 2 \mathrm{GeV})=0.622(69)$ is reasonable compared with those obtained with the WI or $\mathrm{WI}_{\mathrm{VS}}$ method. This linear extrapolation is a credible choice because the chiral behavior of the matrix element $\left\langle\bar{K}^{0}\left|\hat{\mathcal{O}}_{V V+A A}\right| K^{0}\right\rangle$ is linearly recovered as we saw in Fig. 15. We have to make a reservation, however, that this long extrapolation may bring an error larger than quoted in the extrapolated value due to systematic effects of $O\left[a g^{2}(1 / a)\right]$ and $O\left[g^{4}(1 / a)\right]$. The estimation of these systematic errors is too complicated because the matrix elements of the mixing operators have quite different absolute values.

Each of results from the above three methods suffers from statistical and systematic errors of $10-20 \%$ which are comparable in magnitude. Although the $\mathrm{WI}_{\mathrm{Vs}}$ and the PT methods have the advantage of small statistical errors, we recognize that this is offset by the difficulty to control large systematic errors when attempting a continuum extrapolation. We thus conservatively take the result of the WI method $B_{K}(\mathrm{NDR}, 2 \mathrm{GeV})=0.68(7)$ at $a^{-1}=2.7-4.3 \mathrm{GeV}$ as our final estimate of the present work.

\section{CONCLUSIONS}

In this paper we have presented a full account of our method based on chiral Ward identities to nonperturbatively determine the mixing coefficients of the $\Delta s=2$ operator for the Wilson quark action in lattice QCD. Implementing the method in a quenched calculation carried out at four values of lattice spacing, we have demonstrated the effectiveness of the method for constructing the $\Delta s=2$ operator with the correct chiral property. Our final result for $B_{K}(\mathrm{NDR}, 2 \mathrm{GeV})$ $=0.68(7)$ at $a^{-1}=2.7-4.3 \mathrm{GeV}$ shows a reasonable consistency with $B_{K}(\mathrm{NDR}, 2 \mathrm{GeV})=0.628(42)$ in the continuum limit recently obtained with the Kogut-Susskind quark action by us [2].

The error of our Wilson result for $B_{K}$, however, is still too large to convincingly demonstrate that the Wilson and Kogut-Susskind quark actions yield the same value in the continuum limit. We emphasize that this large error is not due to an intrinsic defect of the Ward identity method. It stems from that of large statistical errors of the mixing coefficients, which in turn originates from our use of point source in evaluating relevant quark Green functions. Recent work shows that a variant wall source method with the momentum source for the off-shell quark propagator $[22,24]$ would be effective to diminish the errors of the mixing coefficients.

Another technical point concerns the issue of Gribov copies in the Landau gauge. While an earlier study [25] suggests that ambiguities in the choice of the Gribov copies induce only small uncertainties comparable to typical statistical errors in current numerical simulations, exploring gauge invariant implementation of the Ward identity methods, either employing the external hadron states or the Schrödinger functional, which is free from this problem, would be worthwhile.

In recent calculations of $B_{K}$ using the $O(a)$-improved quark action the chiral property of $\hat{\mathcal{O}}_{V V+A A}$ constructed with one-loop mixing coefficients shows much improvement compared to the Wilson quark case [26]. This observation can be expected on the ground of our perturbative results in the Wilson quark action which suggest in Secs. VI and VII that the leading contribution to chiral breaking effects is $O(a)$. Toward a precise determination of $B_{K}$ the improvement of the quark action is an essential ingredient.

A very important physics issue is the effect of quenching. With the KS quark action it has been observed that the error due to quenched approximation is small $[1,27]$. Whether this is supported by calculations with Wilson action we must defer to future studies. It is straightforward to apply our method once configurations are generated with dynamical quarks.

Finally the application of our method for calculations of $B_{B}$ would be a worthwhile attempt since previous calculations of $B_{B}$ have relied on the mixing coefficients which were calculated perturbatively in the massless limit with tadpole improvement.

\section{ACKNOWLEDGMENTS}

This work was supported by the Supercomputer Project No. 32(FY1998) of the High Energy Accelerator Research Organization (KEK), and also in part by the Grants-in-Aid of the Ministry of Education (Grant Nos. 08640404, 09304029, 10640246, 10640248, 10740107). Y.K. was supported by the Japan Society for Promotion of Science.

\section{APPENDIX}

The continuum renormalization scheme dependence of the renormalization constants $\Delta_{V V+A A}, \Delta_{A}$, and $\Delta_{P}$ defined in Eqs. (54), (60), and (65) have been computed for the NDR, DRED, and RI schemes by a variety of authors $[5,28]$ 
at the one-loop level. We consider it useful to reproduce them in this appendix using off-shell external quark states in the general covariant gauge for quark self-energy and vertex functions.

We consider the following operators:

$$
\begin{aligned}
\mathcal{O}_{\gamma_{\mu}^{L} \otimes \gamma_{\mu}^{L}}= & \frac{\delta_{i j} \delta_{k l}+\delta_{i l} \delta_{k j}}{2} \\
& \times\left(\bar{\psi}_{1}^{i} \gamma_{\mu}\left(1-\gamma_{5}\right) \psi_{2}^{j}\right)\left(\bar{\psi}_{3}^{k} \gamma_{\mu}\left(1-\gamma_{5}\right) \psi_{4}^{l}\right), \\
\mathcal{O}_{\gamma_{\mu} \gamma_{5}}= & \delta_{i j}\left(\bar{\psi}_{1}^{i} \gamma_{\mu} \gamma_{5} \psi_{2}^{j}\right), \\
\mathcal{O}_{\gamma_{5}}= & \delta_{i j}\left(\bar{\psi}_{1}^{i} \gamma_{5} \psi_{2}^{j}\right),
\end{aligned}
$$

where $\gamma_{\mu}^{L} \otimes \gamma_{\mu}^{L}$ represents $\gamma_{\mu}\left(1-\gamma_{5}\right) \otimes \gamma_{\mu}\left(1-\gamma_{5}\right)$ and $i, j$, $k$, and $l$ label the color indices. We note that the operator $\hat{\mathcal{O}}_{V V+A A}$ defined in Eq. (23) is parity conserving part of $\mathcal{O}_{\gamma_{\mu}^{L} \otimes \gamma_{\mu}^{L}}^{L}$ with $\psi_{1}=\psi_{3}=s$ and $\psi_{2}=\psi_{4}=d$.

We draw the relevant one-loop diagrams in Fig. 21; (a) the quark self-energy, (b) the one-loop vertex correction for the quark bilinear operators, and (c)-(h) the six types of the one-loop vertex corrections for the four-quark operator. The off-shell momentum for the external quark state is denoted as $p$. The gauge dependence is parametrized by $\lambda$ expressing the gluon propagator as $\delta_{\mu \nu} / k^{2}-(1-\lambda) k_{\mu} k_{\nu} / k^{4}$.

Up to the one-loop level the inverse quark propagator and the vertex functions for $\Gamma=\gamma_{\mu}^{L} \otimes \gamma_{\mu}^{L}, \gamma_{\mu} \gamma_{5}, \gamma_{5}$ are written in the following form:

$$
\begin{gathered}
G^{-1}(p)=i p-\frac{\alpha_{s}}{4 \pi} \Sigma^{(1)}(p), \\
\Lambda_{\Gamma}(p)=\Gamma+\frac{\alpha_{s}}{4 \pi} \Lambda_{\Gamma}^{(1)}(p),
\end{gathered}
$$

where the superscript (i) refers to the $i$ th loop level. In Table $\mathrm{V}$ we compile the results for $\Sigma^{(1)}$ and $\Lambda_{\Gamma}^{(1)}$ obtained by employing the NDR scheme and the DRED one [29]. The re-
TABLE VI. Scheme dependence of renormalization constants $\Delta_{V V+A A}, \Delta_{A}$, and $\Delta_{P}$.

\begin{tabular}{lcc}
\hline \hline & NDR-DRED & NDR-RI \\
\hline$\Delta_{V V+A A}$ & -3 & $-14 / 3+8 \ln 2$ \\
$\Delta_{A}$ & $-2 / 3$ & 0 \\
$\Delta_{P}$ & $-4 / 3$ & $16 / 3$ \\
\hline \hline
\end{tabular}

duced space-time dimension $D$ is parametrized by $\epsilon$ as $D$ $=4-2 \epsilon, \epsilon>0$. We should note that the one-loop vertex corrections yield the extra evanescent operators which vanish in $D=4$ both for the NDR and the DRED schemes. It is meaningless to give results without mentioning the definition of evanescent operators, because the constant terms at the oneloop level depend on the definition of the evanescent operators. Our choice is as follows:

$$
\begin{aligned}
E_{\gamma_{\mu}^{L} \otimes \gamma_{\mu}^{L}}^{\mathrm{NDR}}= & \frac{1}{4} \gamma_{\rho} \gamma_{\delta} \gamma_{\mu}\left(1-\gamma_{5}\right) \otimes \gamma_{\mu}\left(1-\gamma_{5}\right) \gamma_{\delta} \gamma_{\rho} \\
& -\frac{(2-D)^{2}}{4} \gamma_{\mu}\left(1-\gamma_{5}\right) \otimes \gamma_{\mu}\left(1-\gamma_{5}\right), \\
& E_{\gamma_{\mu} \gamma_{5}}^{\mathrm{DRED}}=\bar{\delta}_{\mu \nu} \gamma_{\nu} \gamma_{5}-\frac{D}{4} \gamma_{\mu} \gamma_{5}, \\
E_{\gamma_{\mu}^{L} \otimes \gamma_{\mu}^{L}}^{\mathrm{DRED}}= & \bar{\delta}_{\mu \nu} \gamma_{\nu}\left(1-\gamma_{5}\right) \otimes \gamma_{\mu}\left(1-\gamma_{5}\right) \\
& -\frac{D}{4} \gamma_{\mu}\left(1-\gamma_{5}\right) \otimes \gamma_{\mu}\left(1-\gamma_{5}\right),
\end{aligned}
$$

where $\bar{\delta}_{\mu \nu}$ is the $D$-dimensional metric tensor.

From the results for $\Sigma^{(1)}(p)$ and $\Lambda_{\Gamma}^{(1)}(p) \quad\left(\Gamma=\gamma_{\mu}^{L}\right.$ $\left.\otimes \gamma_{\mu}^{L}, \gamma_{\mu} \gamma_{5}, \gamma_{5}\right)$ we can extract the scheme dependence of the renormalization constants $\Delta_{V V+A A}, \Delta_{A}$, and $\Delta_{P}$, which are summarized in Table VI. For the RI scheme, for which the renormalization constants depend on the external states and the gauge, we employ the off-shell external quark state with momentum $p^{2}=\mu^{2}$ and the Landau gauge fixing.
[1] G. W. Kilcup et al., Phys. Rev. Lett. 64, 25 (1990); N. Ishizuka et al., ibid. 71, 24 (1993); S. R. Sharpe, Nucl. Phys. B (Proc. Suppl.) 34, 403 (1994).

[2] JLQCD Collaboration, S. Aoki et al., Phys. Rev. Lett. 80, 5271 (1998).

[3] See, e.g., C. Bernard and A. Soni, Nucl. Phys. B (Proc. Suppl.) 9, 155 (1989).

[4] M. B. Gavela et al., Nucl. Phys. B306, 677 (1988); C. Bernard and A. Soni, Nucl. Phys. B (Proc. Suppl.) 42, 391 (1995).

[5] R. Gupta, T. Bhattacharya, and S. Sharpe, Phys. Rev. D 55, 4036 (1997).

[6] G. Martinelli et al., Nucl. Phys. B445, 81 (1995).

[7] A. Donini et al., Phys. Lett. B 360, 83 (1995); M. Crisafulli et al., ibid. 369, 325 (1996); A. Donini et al., Nucl. Phys. B (Proc. Suppl.) 53, 883 (1997); M. Talevi, ibid. 60A, 77 (1998).

[8] M. Bochicchio et al., Nucl. Phys. B262, 331 (1985).

[9] JLQCD Collaboration, S. Aoki et al., Phys. Rev. Lett. 81, 1778 (1998). For preliminary reports, see, JLQCD Collaboration, S. Aoki et al., Nucl. Phys. B (Proc. Suppl.) 53, 349 (1997); 60A, 67 (1998).

[10] L. Maiani and G. Martinelli, Phys. Lett. B 178, 265 (1986).

[11] G. Martinelli, hep-lat/9810013.

[12] G. Martinelli, Phys. Lett. 141B, 395 (1984); C. Bernard, T. Draper, and A. Soni, Phys. Rev. D 36, 3224 (1987).

[13] G. P. Lepage and P. B. Mackenzie, Phys. Rev. D 48, 2250 (1993). 
[14] P. B. Mackenzie, Nucl. Phys. B (Proc. Suppl.) 30, 35 (1993); A. S. Kronfeld, ibid. 30, 445 (1993); A. X. El-Khadra, A. S. Kronfeld, and P. B. Mackenzie, Phys. Rev. D 55, 3933 (1997).

[15] R. Groot, J. Hoek, and J. Smit, Nucl. Phys. B237, 111 (1984).

[16] X. Ji, hep-lat/9506034.

[17] G. Martinelli and Y. C. Zhang, Phys. Lett. 123B, 433 (1983); D. Daniel et al., Phys. Rev. D 46, 3130 (1992).

[18] A. J. Buras and P. H. Weisz, Nucl. Phys. B333, 66 (1990); S. Herrlich and U. Nierste, ibid. B455, 39 (1995).

[19] C. Bernard, T. Draper, G. Hockney, and A. Soni, in Lattice Gauge Theory: A Challenge in Large-Scale Computing, edited by B. Bunk et al. (Plenum, New York, 1986); G. W. Kilcup et al., Phys. Lett. 164B, 347 (1985).

[20] V. Gimenez et al., Nucl. Phys. B531, 429 (1998).
[21] J. R. Cudell, A. Le Yaouanc, and C. Pittori, hep-lat/9810058.

[22] JLQCD Collaboration, S. Aoki et al., hep-lat/9809124.

[23] CP-PACS Collaboration, S. Aoki et al., hep-lat/9809146.

[24] M. Göckeler et al., Nucl. Phys. B (Proc. Suppl.) 63, 868 (1998).

[25] M. L. Paciello, S. Petrarca, B. Taglienti, and A. Vladikas, Phys. Lett. B 341, 187 (1994).

[26] L. Lellouch and C.-J. D. Lin, hep-lat/9809142; R. Gupta, hep-lat/9810016.

[27] G. Kilcup, D. Pekurovsky, and L. Venkataraman, Nucl. Phys. B (Proc. Suppl.) 53, 345 (1997).

[28] M. Ciuchini et al., Z. Phys. C 68, 239 (1995).

[29] G. Altarelli, G. Curci, G. Martinelli, and S. Petrarca, Nucl. Phys. B187, 461 (1981). 